Article

\title{
Bounded Solutions of Semilinear Time Delay Hyperbolic Differential and Difference Equations
}

\author{
Allaberen Ashyralyev 1,2,3,*(i) and Deniz Agirseven 4 \\ 1 Department of Mathematics, Near East University, Mersin 10 99138, Turkey \\ 2 Department of Mathematics, Peoples' Friendship University Russia, Moscow 117198, Russia \\ 3 Institute of Mathematics and Mathematical Modeling, Almaty 050010, Kazakhstan \\ 4 Department of Mathematics, Trakya University, Edirne 22030, Turkey; denizagirseven@trakya.edu.tr or \\ denizagirseven@gmail.com \\ * Correspondence: allaberen.ashyralyev@neu.edu.tr or aallaberen@gmail.com
}

Received: 28 October 2019; Accepted: 27 November 2019; Published: 2 December 2019

\begin{abstract}
In this paper, we study the initial value problem for a semilinear delay hyperbolic equation in Hilbert spaces with a self-adjoint positive definite operator. The mean theorem on the existence and uniqueness of a bounded solution of this differential problem for a semilinear hyperbolic equation with unbounded time delay term is established. In applications, the existence and uniqueness of bounded solutions of four problems for semilinear hyperbolic equations with time delay in unbounded term are obtained. For the approximate solution of this abstract differential problem, the two-step difference scheme of a first order of accuracy is presented. The mean theorem on the existence and uniqueness of a uniformly bounded solution of this difference scheme with respect to time stepsize is established. In applications, the existence and uniqueness of a uniformly bounded solutions with respect to time and space stepsizes of difference schemes for four semilinear partial differential equations with time delay in unbounded term are obtained. In general, it is not possible to get the exact solution of semilinear hyperbolic equations with unbounded time delay term. Therefore, numerical results for the solution of difference schemes for one and two dimensional semilinear hyperbolic equation with time delay are presented. Finally, some numerical examples are given to confirm the theoretical analysis.
\end{abstract}

Keywords: semilinear problems with delay; hyperbolic equations; difference scheme; stability; Hilbert space

MSC: 39A30; 35L20; 65M06

\section{Introduction}

Delay differential equations are used to model biological, physical, system engineering, and sociological processes as well as naturally occurring oscillatory systems (see, for examples, [1-9]).

It is known that in differential and difference equations, the involvement of the delay term causes deep difficulties in the analysis of these equations. $\mathrm{Lu} \mathrm{[10]} \mathrm{studies} \mathrm{modified} \mathrm{iterative} \mathrm{schemes}$ by combing the method of upper-lower solutions and the Jacobi method or the Gauss-Seidel method for finite-difference solutions of reaction-diffusion systems with time delays. Ashyralyev and Sobolevskii [11] study the initial-value problem for linear delay parabolic equations in a Banach space and present a sufficient condition for the stability of the solution of this initial-value problem. The stability estimates in Hölder norms for the solutions of the initial-boundary value problem for delay parabolic equations were established.

Ashyralyev and Agirseven [12-18] studied some initial-boundary value problems for linear delay parabolic differential equations. Theorems on stability and convergence of difference schemes for the 
numerical solution of initial and boundary value problems for linear parabolic equations with time delay were proved. Moreover, Ashyralyev, Agirseven, and Ceylan [19] investigated finding sufficient conditions for the existence and uniqueness of a bounded solution of the initial value problem for the semilinear delay parabolic equation in a Banach space. The main theorem on the existence and uniqueness of a bounded solution of this problem was established. In applications, existence and uniqueness of a bounded solution of problems for four semilinear delay parabolic equations were obtained. Numerical results were given.

Henriquez, Cuevas, and Caicedo [20] investigated the existence of almost periodic solutions for linear retarded functional differential equations with finite delay. The existence of almost periodic solutions with the stabilization of distributed control systems was obtained.

Hao, Fan, Cao, and Sun [21] proposed a linearized quasi-compact finite difference scheme for semilinear space-fractional diffusion equations with a fixed time delay. Under the local Lipschitz conditions, they proved the solvability and convergence of the scheme in the discrete maximum norm by the energy method.

Liang [22] studied the convergence and asymptotic stability of semidiscrete and full discrete schemes for linear parabolic equations with time delay. She proved that the semidiscrete scheme, backward Euler and Crank-Nicolson full discrete schemes can unconditionally preserve the delay-independent asymptotic stability with some additional restrictions on time and spatial stepsizes of the forward Euler full discrete scheme.

Bhrawy, Abdelkawy, and Mallawi [23] investigated the Chebyshev Gauss-Lobatto pseudospectral scheme in spatial directions for the approximate solution of one-dimensional, coupled, and two-dimensional parabolic equations with time delays. They also develop an efficient numerical algorithm based on the Chebyshev pseudospectral algorithm to obtain the two spatial variables in approximate solving the two-dimensional parabolic equations with time delay.

Applying Vishik's results and methods of operator tools, Ismailov, Guler, and Ipek [24] described all solvable extensions of a minimal operator generated by linear delay differential-operator expression of first order in the Hilbert space of vector-functions in finite interval. Sharp formulas for the spectrums of these solvable extensions were obtained. Theoretical results have been supported by applications.

Piriadarshani and Sengadir [25] obtained an existence theorem for a semilinear partial differential equation with infinite delay employing a phase space in which discretizations can naturally be performed. For linear partial differential equations with infinite delay they show that the solutions of the ordinary differential equation with infinite delay obtained by the semi-discretization converge to the original solution.

Castro, Rodriguez, Cabrera, and Martin [26] developed an explicit finite difference scheme for a model with coefficients variable in time and studied their properties of convergence and stability.

Hyperbolic equations without time delay arise in many branches of science and engineering, for example, electrodynamics, thermodynamics, hydrodynamics, fluid dynamics, wave propagation, hyperbolic geometry, and discrete mathematics (see, e.g., [8,9,27-34], and the references given therein). The geometry of complex networks is closely related with their structure and function. Shang Yilun [34] investigated the Gromov-hyperbolicity of the Newman-Watts model of small-world networks. It is known that asymptotic Erdős-Rényi random graphs are not hyperbolic. We show that the Newman-Watts ones built on top of them by adding lattice-induced clustering are not hyperbolic as the network size goes to infinity. Numerical simulations are provided to illustrate the effects of various parameters on hyperbolicity in this model. The geometry of complex networks has a close relationship with their structure and function. Shang Yilun [33] investigated Gromov-hyperbolicity of inhomogeneous random networks modeled by the Chung-Lu model $G(w)$. His numerical simulations illustrate this non-hyperbolicity of $G(w)$ for power law degree distributions among others.

In numerical methods for solving hyperbolic equations, the problem of stability has received a great deal of importance and attention. The method of operators as a tool for the study of the stability of 
the solution of local and nonlocal problems to hyperbolic differential and difference equations in Hilbert and Banach spaces has been systematically developed by many authors (see, e.g., [27-32,35-37]).

A large cycle of works on difference schemes for hyperbolic equations (see, e.g., [38-42] and the references given therein), in which stability was established under the assumption that the magnitude of the grid steps $\tau$ and $h$ with respect to the time and space variables, were connected. In abstract terms that means that the condition $\tau\left\|A_{h}\right\| \rightarrow 0$ when $\tau \rightarrow 0$ holds.

Of course there is great interest in the study of absolute stable difference schemes of a high order of accuracy for hyperbolic equations, in which stability was established without any assumptions in respect to the grid steps $\tau$ and $h$. Such type of stability inequalities for the solutions of the first order of accuracy difference scheme for the abstract hyperbolic equation in Hilbert spaces were established for the first time in [43]. The first and second order of accuracy difference schemes generated by integer power of space operator of approximate solutions of the abstract initial value problem for the abstract hyperbolic equation in Hilbert spaces were presented in [11]. The stability estimates for the solution of these difference schemes were established.

The survey paper [44] contains the recent results on the local and nonlocal well-posed problems for second order differential and difference equations. Stability of differential problems for hyperbolic equations and of difference schemes for approximate solution of these problems were presented.

However, the stability theory of problems for a hyperbolic equation with unbounded time delay term is not well-investigated. A few researchers are interested in these kinds of problems. Bounded solutions of semilinear one dimensional hyperbolic differential equations with time delay term have been investigated in earlier papers [45-48]. In the paper [49] the existence and uniqueness of a bounded solution of nonlinear hyperbolic differential equations with bounded time delay term were established. The generality of the operator approach allows for treating a wider class of delay nonlinear hyperbolic differential equations with bounded time delay term. In general, hyperbolic differential equations with unbounded time delay term are blown up [7]. Therefore, the boundedness solution of problems for hyperbolic equations with unbounded time delay term is not well-investigated.

Our goal in the present paper is to investigate the boundedness solution of problems for semilinear hyperbolic equations with unbounded time delay term. We study the initial value problem for the semilinear hyperbolic differential equation with time delay

$$
\left\{\begin{array}{l}
\frac{d^{2} u(t)}{d t^{2}}+A u(t)=f\left(t, u(t), u_{t}(t-w), u(t-w)\right), t>0 \\
u(t)=\varphi(t),-\omega \leq t \leq 0
\end{array}\right.
$$

in a Hilbert space $H$ with a self-adjoint positive definite operator $A$. Here $\varphi(t)$ is a continuously differentiable abstract function defined on the interval $[-\omega, 0]$ with values in $H$ and $f(t)$ is continuous abstract function defined on the interval $[0, \infty)$ with values in $H$. Assume that $A$ is unbounded operator and $(A x, x)>\delta(x, x)$, for $x \neq 0, x \in H$ and $\delta>0$.

A function $u(t)$ is called a solution of problem (1), if the following conditions are fulfilled:

i. $u(t)$ is twice continuously differentiable function on the interval $[0, \infty), f\left(t, u(t), u_{t}(t-w), u(t-\right.$ $w)$ ) is continuous and bounded function on $[0, \infty)$

ii. The element $u(t)$ belongs to $D(A)$ for all $t \in[0, \infty)$, and the function $A u(t)$ is continuous on the interval $[0, \infty)$.

iii. $u(t)$ satisfies the equation and initial conditions in Equation (1).

In the present paper, the main theorem on the existence and uniqueness of a bounded solution of the differential problem (1) is established. In applications, the existence and uniqueness of a bounded solution of four problems for semilinear hyperbolic equations with time delay are obtained. A first order of accuracy difference scheme for the numerical solution of this problem is presented. The theorem on the existence and uniqueness of a uniformly bounded solution of this difference scheme 
with respect to $\tau$ is established. In applications, existence and uniqueness of a bounded solution of a problem for four semilinear delay parabolic equations were established. Numerical results for the solution of difference schemes for one and two dimensional nonlinear hyperbolic equation with time delay are presented.

\section{Main Existence and Uniqueness Theorem of the Differential Problem}

Throughout this paper, $c(t)$ and $s(t)$ are operator-functions defined by formulas

$$
c(t) u=\frac{e^{i A^{\frac{1}{2}} t}+e^{-i A^{\frac{1}{2}} t}}{2} u, s(t) u=\int_{0}^{t} c(y) u d y .
$$

We will give some auxiliary statements which will be useful in the sequel.

Recall that the norm $\|A\|_{H \rightarrow H}$ of a bounded operator $A: H \rightarrow H$ is by definition the smallest number $C$ for which estimate

$$
\|A u\|_{H} \leq C\|u\|_{H}
$$

holds. Equivalently,

$$
\|A\|_{H \rightarrow H}=\sup _{\|u\|_{H}=1}\|A u\|_{H} .
$$

Lemma 1. For $t \geq 0$, the following estimates hold:

$$
\left\|A^{-\frac{1}{2}}\right\|_{H \rightarrow H} \leq \delta^{-\frac{1}{2}},\|c(t)\|_{H \rightarrow H} \leq 1,\|A s(t)\|_{H \rightarrow H} \leq 1 .
$$

Proof. Applying formulas (2) and the spectral representation of the self-adjoint positive definite operator $A$ in a Hilbert space $H$, we can write (see [50])

$$
\begin{gathered}
\left\|A^{-\frac{1}{2}}\right\|_{H \rightarrow H} \leq \sup _{\delta \leq \lambda<\infty}\left|\lambda^{-\frac{1}{2}}\right| \leq \delta^{-\frac{1}{2}}, \\
\|c(t)\|_{H \rightarrow H} \leq \sup _{\delta \leq \lambda<\infty}\left|\frac{e^{i \lambda^{\frac{1}{2}} t}+e^{-i \lambda^{\frac{1}{2}} t}}{2}\right|=\sup _{\delta \leq \lambda<\infty}\left|\cos \left(\lambda^{\frac{1}{2}} t\right)\right| \leq 1, \\
\left\|A^{\frac{1}{2}} s(t)\right\|_{H \rightarrow H} \leq \sup _{\delta \leq \lambda<\infty}\left|\frac{e^{i \lambda^{\frac{1}{2}} t}-e^{-i \lambda^{\frac{1}{2}} t}}{2 i}\right|=\sup _{\delta \leq \lambda<\infty}\left|\sin \left(\lambda^{\frac{1}{2}} t\right)\right| \leq 1,
\end{gathered}
$$

for any $t \geq 0$. Lemma 1 is proved.

The approach of proof of main theorem is based on reducing problem (1) to an integral equation of Volterra type

$$
\begin{aligned}
u(t)= & c(t-(m-1) w) u((m-1) w)+s(t-(m-1) w) \frac{d u((m-1) w)}{d t} \\
& +\int_{(m-1) w}^{t} s(t-y) f\left(y, u(y), u_{y}(y-w), u(y-w)\right) d y \\
(m-1) w \leq t \leq m w, & m=1,2, \ldots, u(t)=\varphi(t),-w \leq t \leq 0
\end{aligned}
$$

in $[0, \infty) \times H$ and the application of successive approximations. Note that on $(m-1) w \leq t \leq m w$, $m=1,2, \ldots, u_{t}(t-w)$ and $u(t-w)$ are given. Therefore, the recursive formula for the solution of 
problem (1) is

$$
\begin{gathered}
u_{i}(t)=\begin{array}{c}
c(t-(m-1) w) u((m-1) w)+s(t-(m-1) w) \frac{d u((m-1) w)}{d t} \\
+\int_{(m-1) w}^{t} s(t-y) f\left(y, u_{i-1}(y), u_{y}(y-w), u(y-w)\right) d y
\end{array} \\
u_{0}(t)=c(t-(m-1) w) u((m-1) w)+s(t-(m-1) w) \frac{d u((m-1) w)}{d t}, \\
(m-1) w \leq t \leq m w, m=1,2, \ldots, \\
u_{i}(t)=\varphi(t), i=1,2, \ldots,-w \leq t \leq 0 .
\end{gathered}
$$

Theorem 1. Suppose that $\varphi(t)$ is a continuously differentiable function on $[-\omega, 0]$ and $\varphi(t) \in D(A)$, $\varphi^{\prime}(t) \in D\left(A^{\frac{1}{2}}\right)$ and

$$
\|A \varphi(t)\|_{H} \leq M,\left\|A^{1 / 2} \varphi^{\prime}(t)\right\|_{H} \leq \tilde{M}
$$

Besides let $f:[0, \infty) \times H \times H \times H \longrightarrow H$ be continuous and bounded function, that is

$$
\|f(t, u, v, z)\|_{H} \leq \bar{M}
$$

in $[0, \infty) \times H \times H \times H$ and Lipschitz condition holds uniformly with respect to $t, v$ and $z$

$$
\|f(t, u, v, z)-f(t, w, v, z)\|_{H} \leq L\|u-w\|_{H}
$$

Here, $M, \widetilde{M}, \bar{M}, \mathrm{七}$ are positive constants. Then there exists a unique solution to problem (1) which is bounded in $\mathrm{C}(H)$. Here, $\mathrm{C}(H)=\mathrm{C}([0, \infty), H)$ stands for the Banach space of the abstract continuous and bounded functions $v(t)$ defined on $[0, \infty)$ with values in $H$, equipped with the norm

$$
\|v\|_{C(H)}=\sup _{0 \leq t<\infty}\|v(t)\|_{H}
$$

Proof. Let $0 \leq t \leq \omega$. Then, according to Equation (4), we get

$$
\begin{gathered}
u_{i}(t)=c(t) \varphi(0)+s(t) \varphi^{\prime}(0)+\int_{0}^{t} s(t-y) f\left(y, u_{i-1}(y), \varphi_{y}(y-w), \varphi(y-w)\right) d y, \\
u_{i}^{\prime}(t)=-A s(t) \varphi(0)+c(t) \varphi^{\prime}(0)+\int_{0}^{t} c(t-y) f\left(y, u_{i-1}(y), \varphi_{y}(y-w), \varphi(y-w)\right) d y
\end{gathered}
$$

for all $i=1,2, \ldots$. Therefore,

$$
\begin{aligned}
& u(t)=u_{0}(t)+\sum_{i=0}^{\infty}\left(u_{i+1}(t)-u_{i}(t)\right), \\
& u^{\prime}(t)=u_{0}^{\prime}(t)+\sum_{i=0}^{\infty}\left(u_{i+1}^{\prime}(t)-u_{i}^{\prime}(t)\right),
\end{aligned}
$$

where

$$
u_{0}(t)=c(t) \varphi(0)+s(t) \varphi^{\prime}(0), u_{0}^{\prime}(t)=-A s(t) \varphi(0)+c(t) \varphi^{\prime}(0) .
$$

Applying the triangle inequality and estimates (3) and (5), we get

$$
\begin{gathered}
\left\|u_{0}(t)\right\|_{H} \leq\left\|A^{-1}\right\|_{H \rightarrow H} \\
\times\left[\|c(t)\|_{H \rightarrow H}\|A \varphi(0)\|_{H}+\left\|A^{1 / 2} s(t)\right\|_{H \rightarrow H}\left\|A^{1 / 2} \varphi^{\prime}(0)\right\|_{H}\right] \\
\leq \delta^{-1}\left[\|A \varphi(0)\|_{H}+\left\|A^{1 / 2} \varphi^{\prime}(0)\right\|_{H}\right] \leq \delta^{-1}[M+\tilde{M}] \\
\left\|u_{0}^{\prime}(t)\right\|_{H} \leq\left\|A^{-\frac{1}{2}}\right\|_{H \rightarrow H}
\end{gathered}
$$




$$
\begin{gathered}
\times\left[\left\|A^{1 / 2} s(t)\right\|_{H \rightarrow H}\|A \varphi(0)\|_{H}+\|c(t)\|_{H \rightarrow H}\left\|A^{1 / 2} \varphi^{\prime}(0)\right\|_{H}\right] \\
\leq \delta^{-\frac{1}{2}}\left[\|A \varphi(0)\|_{H}+\left\|A^{1 / 2} \varphi^{\prime}(0)\right\|_{H}\right] \leq \delta^{-\frac{1}{2}}[M+\widetilde{M}] .
\end{gathered}
$$

Applying formulas (8) and (9) and the triangle inequality and estimates (3) and (6), we get

$$
\begin{gathered}
\left\|u_{1}(t)-u_{0}(t)\right\|_{H} \leq\left\|A^{-\frac{1}{2}}\right\|_{H \rightarrow H} \\
\times \int_{0}^{t}\left\|A^{1 / 2} S(t-y)\right\|_{H \rightarrow H}\left\|f\left(y, u_{0}(y), \varphi_{y}(y-w), \varphi(y-w)\right)\right\|_{H} d y \leq \delta^{-\frac{1}{2}} \bar{M} t \\
\left\|u_{1}^{\prime}(t)-u_{0}^{\prime}(t)\right\|_{H} \leq \int_{0}^{t}\|c(t-y)\|_{H \rightarrow H}\left\|f\left(y, u_{0}(y), \varphi_{y}(y-w), \varphi(y-w)\right)\right\|_{H} d y \leq \bar{M} t .
\end{gathered}
$$

Using the triangle inequality, we get

$$
\begin{gathered}
\left\|u_{1}(t)\right\|_{H} \leq \delta^{-1}[M+\tilde{M}]+\delta^{-\frac{1}{2}} \bar{M} t \\
\left\|u_{1}^{\prime}(t)\right\|_{H} \leq \delta^{-\frac{1}{2}}[M+\tilde{M}]+\bar{M} t
\end{gathered}
$$

Applying formulas (8) and (9) and estimates (3), (6), and (7), we get

$$
\left\|u_{2}(t)-u_{1}(t)\right\|_{H} \leq\left\|A^{-\frac{1}{2}}\right\|_{H \rightarrow H}
$$

$\times \int_{0}^{t}\left\|A^{1 / 2} s(t-y)\right\|_{H \rightarrow H}\left\|f\left(y, u_{1}(y), \varphi_{y}(y-w), \varphi(y-w)\right)-f\left(y, u_{0}(y), \varphi_{y}(y-w), \varphi(y-w)\right)\right\|_{H} d y$

$$
\begin{gathered}
\leq \delta^{-\frac{1}{2}} L \int_{0}^{t}\left\|u_{1}(y)-u_{0}(y)\right\|_{H} d y \leq \delta^{-1} L \bar{M} \int_{0}^{t} y d y=\frac{\bar{M}}{L} \frac{\left(\delta^{-\frac{1}{2}} L t\right)^{2}}{2 !}, \\
\left\|u_{2}^{\prime}(t)-u_{1}^{\prime}(t)\right\|_{H}
\end{gathered}
$$

$\leq \int_{0}^{t}\|c(t-y)\|_{H \rightarrow H}\left\|f\left(y, u_{1}(y), \varphi_{y}(y-w), \varphi(y-w)\right)-f\left(y, u_{0}(y), \varphi_{y}(y-w), \varphi(y-w)\right)\right\|_{H} d y$

$$
\leq L \int_{0}^{t}\left\|u_{1}(y)-u_{0}(y)\right\|_{H} d y \leq \delta^{-\frac{1}{2}} L \bar{M} \int_{0}^{t} y d y=\delta^{-\frac{1}{2}} \frac{\bar{M}}{L} \frac{(L t)^{2}}{2 !} .
$$

Then

$$
\begin{gathered}
\left\|u_{2}(t)\right\|_{H} \leq \delta^{-1}[M+\tilde{M}]+\delta^{-\frac{1}{2}} \bar{M} t+\frac{\bar{M}}{L} \frac{\left(\delta^{-\frac{1}{2}} L t\right)^{2}}{2 !}, \\
\left\|u_{2}^{\prime}(t)\right\|_{H} \leq \delta^{-\frac{1}{2}}[M+\tilde{M}]+\bar{M} t+\frac{\bar{M}}{L \delta^{-\frac{1}{2}}} \frac{(L t)^{2}}{2 !} .
\end{gathered}
$$

Let

$$
\left\|u_{n}(t)-u_{n-1}(t)\right\|_{H} \leq \frac{\bar{M}}{L} \frac{\left(\delta^{-\frac{1}{2}} L t\right)^{n}}{n !},\left\|u_{n}^{\prime}(t)-u_{n-1}^{\prime}(t)\right\|_{H} \leq \frac{\bar{M}}{L \delta^{-\frac{1}{2}}} \frac{(L t)^{n}}{n !}
$$

and

$$
\begin{gathered}
\left\|u_{n}(t)\right\|_{H} \leq \delta^{-1}[M+\tilde{M}]+\delta^{-\frac{1}{2}} \bar{M} t+\frac{\bar{M}}{L} \frac{\left(\delta^{-\frac{1}{2}} L t\right)^{2}}{2 !}+\cdots+\frac{\bar{M}}{L} \frac{\left(\delta^{-\frac{1}{2}} L t\right)^{n}}{n !}, \\
\left\|u_{n}^{\prime}(t)\right\|_{H} \leq \delta^{-\frac{1}{2}}[M+\tilde{M}]+\bar{M} t+\frac{\bar{M}}{L \delta^{-\frac{1}{2}}} \frac{\left(L \delta^{-\frac{1}{2}} t\right)^{2}}{2 !}+\cdots+\frac{\bar{M}}{L \delta^{-\frac{1}{2}}} \frac{\left(L \delta^{-\frac{1}{2}} t\right)^{n}}{n !} .
\end{gathered}
$$

Then, we obtain

$$
\left\|u_{n+1}(t)-u_{n}(t)\right\|_{H} \leq\left\|A^{-\frac{1}{2}}\right\|_{H \rightarrow H}
$$


$\times \int_{0}^{t}\left\|A^{1 / 2} s(t-y)\right\|_{H \rightarrow H}\left\|f\left(y, u_{n}(y), \varphi_{y}(y-w), \varphi(y-w)\right)-f\left(y, u_{n-1}(y), \varphi_{y}(y-w), \varphi(y-w)\right)\right\|_{H} d y$ $\leq \delta^{-\frac{1}{2}} L \int_{0}^{t}\left\|u_{n}(y)-u_{n-1}(y)\right\|_{H} d y \leq \delta^{-\frac{1}{2}} L \int_{0}^{t} \frac{\bar{M}}{L} \frac{\left(\delta^{-\frac{1}{2}} L y\right)^{n}}{n !} y d y=\frac{\bar{M}}{L} \frac{\left(\delta^{-\frac{1}{2}} L t\right)^{n+1}}{(n+1) !}$

$$
\left\|u_{n+1}^{\prime}(t)-u_{n}^{\prime}(t)\right\|_{H}
$$

$\leq \int_{0}^{t}\|c(t-y)\|_{H \rightarrow H}\left\|f\left(y, u_{n}(y), \varphi_{y}(y-w), \varphi(y-w)\right)-f\left(y, u_{n-1}(y), \varphi_{y}(y-w), \varphi(y-w)\right)\right\|_{H} d y$

$$
\leq \int_{0}^{t} L\left\|u_{n}(y)-u_{n-1}(y)\right\|_{H} d s \leq \int_{0}^{t} L \frac{\bar{M}}{L} \frac{\left(\delta^{-\frac{1}{2}} L y\right)^{n}}{n !} d y=\frac{\bar{M}}{L \delta^{-\frac{1}{2}}} \frac{\left(L \delta^{-\frac{1}{2}} t\right)^{n+1}}{(n+1) !} .
$$

Therefore,

$$
\left\|u_{n+1}(t)-u_{n}(t)\right\|_{H} \leq \frac{\bar{M}}{L} \frac{\left(\delta^{-\frac{1}{2}} L t\right)^{n+1}}{(n+1) !},\left\|u_{n+1}^{\prime}(t)-u_{n}^{\prime}(t)\right\|_{H} \leq \frac{\bar{M}}{L \delta^{-\frac{1}{2}}} \frac{\left(L \delta^{-\frac{1}{2}} t\right)^{n+1}}{(n+1) !}
$$

and

$$
\begin{gathered}
\left\|u_{n+1}(t)\right\|_{H} \leq \delta^{-1}[M+\tilde{M}]+\delta^{-\frac{1}{2}} \bar{M} t+\frac{\bar{M}}{L} \frac{\left(\delta^{-\frac{1}{2}} L t\right)^{2}}{2 !} \cdots+\frac{\bar{M}}{L} \frac{\left(\delta^{-\frac{1}{2}} L t\right)^{n+1}}{(n+1) !} \\
\left\|u_{n+1}^{\prime}(t)\right\|_{H} \leq \delta^{-\frac{1}{2}}[M+\tilde{M}]+\bar{M} t+\frac{\bar{M}}{L \delta^{-\frac{1}{2}}} \frac{\left(L \delta^{-\frac{1}{2}} t\right)^{2}}{2 !}+\cdots+\frac{\bar{M}}{L \delta^{-\frac{1}{2}}} \frac{\left(L \delta^{-\frac{1}{2}} t\right)^{n+1}}{(n+1) !}
\end{gathered}
$$

are true for any $n, n \geq 1$ by mathematical induction. In a similar manner, for any $n$, we can obtain

$$
\left\|A^{\frac{1}{2}} u_{n+1}(t)-A^{\frac{1}{2}} u_{n}(t)\right\|_{H} \leq \frac{\bar{M}}{L \delta^{-\frac{1}{2}}} \frac{\left(L \delta^{-\frac{1}{2}} t\right)^{n+1}}{(n+1) !}
$$

and

$$
\left\|A^{\frac{1}{2}} u_{n+1}(t)\right\|_{H} \leq \delta^{-\frac{1}{2}}[M+\tilde{M}]+\bar{M} t+\frac{\bar{M}}{L \delta^{-\frac{1}{2}}} \frac{\left(L \delta^{-\frac{1}{2}} t\right)^{2}}{2 !}+\cdots+\frac{\bar{M}}{L \delta^{-\frac{1}{2}}} \frac{\left(L \delta^{-\frac{1}{2}} t\right)^{n+1}}{(n+1) !} .
$$

From that and formulas (10) and (11) it follows that

$$
\begin{gathered}
\|u(t)\|_{H} \leq\left\|u_{0}(t)\right\|_{H}+\sum_{i=0}^{\infty}\left\|u_{i+1}(t)-u_{i}(t)\right\|_{H} \\
\leq \delta^{-1}[M+\tilde{M}]+\sum_{i=0}^{\infty} \frac{\bar{M}}{L} \frac{\left(\delta^{-\frac{1}{2}} L t\right)^{i+1}}{(i+1) !} \\
\leq \delta^{-1}[M+\tilde{M}]+\frac{\bar{M}}{L} e^{\delta^{-\frac{1}{2}} L t}, 0 \leq t \leq w \\
\left\|u^{\prime}(t)\right\|_{H} \leq\left\|u_{0}^{\prime}(t)\right\|_{H}+\sum_{i=0}^{\infty}\left\|u_{i+1}^{\prime}(t)-u_{i}^{\prime}(t)\right\|_{H} \\
\leq \delta^{-\frac{1}{2}}[M+\tilde{M}]+\sum_{i=0}^{\infty} \frac{\bar{M}}{L \delta^{-\frac{1}{2}}} \frac{\left(L \delta^{-\frac{1}{2}} t\right)^{i+1}}{(i+1) !} \\
\leq \delta^{-\frac{1}{2}}[M+\tilde{M}]+\frac{\bar{M}}{L \delta^{-\frac{1}{2}}} e^{L \delta^{-\frac{1}{2}} t}, 0 \leq t \leq w \\
\left\|A^{\frac{1}{2}} u(t)\right\|_{H} \leq\left\|A^{\frac{1}{2}} u_{0}(t)\right\|_{H}+\sum_{i=0}^{\infty}\left\|A^{\frac{1}{2}} u_{i+1}(t)-A^{\frac{1}{2}} u_{i}(t)\right\|_{H}
\end{gathered}
$$




$$
\begin{aligned}
& \leq \delta^{-\frac{1}{2}}[M+\tilde{M}]+\sum_{i=0}^{\infty} \frac{\bar{M}}{L \delta^{-\frac{1}{2}}} \frac{\left(L \delta^{-\frac{1}{2}} t\right)^{i+1}}{(i+1) !} \\
\leq & \delta^{-\frac{1}{2}}[M+\tilde{M}]+\frac{\bar{M}}{L \delta^{-\frac{1}{2}}} e^{L \delta^{-\frac{1}{2}} t}, 0 \leq t \leq w,
\end{aligned}
$$

which proves the existence of a bounded solution of problem (1) in $[0, w] \times H$.

Let $m \omega \leq t \leq(m+1) \omega, m=1,2, \ldots$. Then, according to Equation (4), we can write

$$
\begin{gathered}
u_{i}(t)=c(t-m w) u(m w)+s(t-m w) \frac{d u(m w)}{d t} \\
+\int_{m w}^{t} s(t-y) f\left(y, u_{i-1}(y), u_{y}(y-w), u(y-w)\right) d y, \quad i=1,2, \ldots \\
u_{i}^{\prime}(t)=-A s(t-m w) u(m w)+c(t-m w) \frac{d u(m w)}{d t} \\
+\int_{m w}^{t} c(t-y) f\left(y, u_{i-1}(y), u_{y}(y-w), u(y-w)\right) d y, \quad i=1,2, \ldots
\end{gathered}
$$

Therefore,

$$
\begin{aligned}
& u(t)=u_{0}(t)+\sum_{i=0}^{\infty}\left(u_{i+1}(t)-u_{i}(t)\right), \\
& u_{i}^{\prime}(t)=u_{0}^{\prime}(t)+\sum_{i=0}^{\infty}\left(u_{i+1}^{\prime}(t)-u_{i}^{\prime}(t)\right),
\end{aligned}
$$

where

$$
\begin{gathered}
u_{0}(t)=c(t-m w) u(m w)+s(t-m w) \frac{d u(m w)}{d t} \\
u_{0}^{\prime}(t)=-A s(t-m w) u(m w)+c(t-m w) \frac{d u(m w)}{d t} .
\end{gathered}
$$

Assume that problem (1) has a bounded solution in $[(m-1) \omega, m w] \times H$ and

$$
\left\|A^{1 / 2} u(t)\right\|_{H} \leq M_{m-1},\left\|u^{\prime}(t)\right\|_{H} \leq \widetilde{M}_{m-1} .
$$

Applying estimates (3) and (16), we get

$$
\begin{gathered}
\left\|u_{0}(t)\right\|_{H} \leq\left\|A^{-\frac{1}{2}}\right\|_{H \rightarrow H} \\
\times\left[\|c(t)\|_{H \rightarrow H}\left\|A^{1 / 2} u(m w)\right\|_{H}+\left\|A^{1 / 2} s(t)\right\|_{H \rightarrow H}\left\|u^{\prime}(m w)\right\|_{H}\right] \leq \delta^{-\frac{1}{2}}\left[M_{m-1}+\widetilde{M}_{m-1}\right], \\
\left\|u_{0}^{\prime}(t)\right\|_{H} \leq\left[\left\|A^{1 / 2} s(t)\right\|_{H \rightarrow H}\left\|A^{1 / 2} u(m w)\right\|_{H}+\|c(t)\|_{H \rightarrow H}\left\|\varphi^{\prime}(0)\right\|_{H}\right] \leq M_{m-1}+\tilde{M}_{m-1} .
\end{gathered}
$$

Applying formulas (12) and (13) and estimates (3) and (6), we get

$$
\begin{gathered}
\left\|u_{1}(t)-u_{0}(t)\right\|_{H} \leq\left\|A^{-\frac{1}{2}}\right\|_{H \rightarrow H} \\
\times \int_{m \omega}^{t}\left\|A^{1 / 2} s(t-y)\right\|_{H \rightarrow H}\left\|f\left(y, u_{0}(y), u_{y}(y-w), u(y-w)\right)\right\|_{H} d y \leq \delta^{-\frac{1}{2}} \bar{M}(t-m \omega), \\
\left\|u_{1}^{\prime}(t)-u_{0}^{\prime}(t)\right\|_{H} \leq \int_{m \omega}^{t}\|c(t-y)\|_{H \rightarrow H}\left\|f\left(y, u_{0}(y), u_{y}(y-w), u(y-w)\right)\right\|_{H} d y \leq \bar{M}(t-m \omega) .
\end{gathered}
$$

Using the triangle inequality, we get

$$
\begin{gathered}
\left\|u_{1}(t)\right\|_{H} \leq \delta^{-\frac{1}{2}}\left[M_{m-1}+\tilde{M}_{m-1}\right]+\delta^{-\frac{1}{2}} \bar{M}(t-m \omega), \\
\left\|u_{1}^{\prime}(t)\right\|_{H} \leq M_{m-1}+\widetilde{M}_{m-1}+\bar{M}(t-m \omega) .
\end{gathered}
$$


Applying formulas (9) and (12) and estimates (3), (6), and (7), we get

$$
\begin{gathered}
\left\|u_{2}(t)-u_{1}(t)\right\|_{H} \leq\left\|A^{-\frac{1}{2}}\right\|_{H \rightarrow H} \\
\times \int_{0}^{t}\left\|A^{1 / 2} s(t-y)\right\|_{H \rightarrow H}\left\|f\left(y, u_{1}(y), u_{y}(y-w), u(y-w)\right)-f\left(y, u_{0}(y), u_{y}(y-w), u(y-w)\right)\right\|_{H} d y \\
\leq \delta^{-\frac{1}{2}} L \int_{m \omega}^{t}\left\|u_{1}(y)-u_{0}(y)\right\|_{H} d y \leq \delta^{-1} L \bar{M} \int_{m \omega}^{t}(y-m \omega) d y=\frac{\bar{M}}{L} \frac{\left(\delta^{-\frac{1}{2}} L(t-m \omega)\right)^{2}}{2 !}, \\
\left\|u_{2}^{\prime}(t)-u_{1}^{\prime}(t)\right\|_{H} \\
\leq \int_{m \omega}^{t}\|c(t-y)\|_{H \rightarrow H}\left\|f\left(y, u_{1}(y), u_{y}(y-w), u(y-w)\right)-f\left(y, u_{0}(y), u_{y}(y-w), u(y-w)\right)\right\|_{H} d y \\
\leq L \int_{m \omega}^{t}\left\|u_{1}(y)-u_{0}(y)\right\|_{H} d y \leq L \bar{M} \delta^{-\frac{1}{2}} \int_{m \omega}^{t}(y-m \omega) d y=\frac{\bar{M}}{L \delta^{-\frac{1}{2}}} \frac{\left(L \delta^{-\frac{1}{2}}(t-m \omega)\right)^{2}}{2 !} .
\end{gathered}
$$

Then

$$
\begin{gathered}
\left\|u_{2}(t)\right\|_{H} \leq \delta^{-\frac{1}{2}}\left[M_{m-1}+\widetilde{M}_{m-1}\right]+\delta^{-\frac{1}{2}} \bar{M}(t-m \omega)+\frac{\bar{M}}{L} \frac{\left(\delta^{-\frac{1}{2}} L(t-m \omega)\right)^{2}}{2 !} \\
\left\|u_{2}^{\prime}(t)\right\|_{H} \leq M_{m-1}+\widetilde{M}_{m-1}+\bar{M}(t-m \omega)+\frac{\bar{M}}{L \delta^{-\frac{1}{2}}} \frac{\left(L \delta^{-\frac{1}{2}}(t-m \omega)\right)^{2}}{2 !} .
\end{gathered}
$$

Let

$$
\left\|u_{n}(t)-u_{n-1}(t)\right\|_{H} \leq \frac{\bar{M}}{L} \frac{\left(\delta^{-\frac{1}{2}} L(t-m \omega)\right)^{n}}{n !},\left\|u_{n}^{\prime}(t)-u_{n-1}^{\prime}(t)\right\|_{H} \leq \frac{\bar{M}}{L \delta^{-\frac{1}{2}}} \frac{\left(L \delta^{-\frac{1}{2}}(t-m \omega)\right)^{n}}{n !}
$$

and

$$
\begin{gathered}
\left\|u_{n}(t)\right\|_{H} \leq \delta^{-\frac{1}{2}}\left[M_{m-1}+\widetilde{M}_{m-1}\right]+\delta^{-\frac{1}{2}} \bar{M}(t-m \omega)+\frac{\bar{M}}{L} \frac{\left(\delta^{-\frac{1}{2}} L(t-m \omega)\right)^{2}}{2 !}+\cdots+\frac{\bar{M}}{L} \frac{\left(\delta^{-\frac{1}{2}} L(t-m \omega)\right)^{n}}{n !}, \\
\left\|u_{n}^{\prime}(t)\right\|_{H} \leq M_{m-1}+\widetilde{M}_{m-1}+\bar{M}(t-m \omega)+\frac{\bar{M}}{L \delta^{-\frac{1}{2}}} \frac{\left(L \delta^{-\frac{1}{2}}(t-m \omega)\right)^{2}}{2 !}+\cdots+\frac{\bar{M}}{L \delta^{-\frac{1}{2}}} \frac{\left(L \delta^{-\frac{1}{2}}(t-m \omega)\right)^{n}}{n !} .
\end{gathered}
$$

Then, we obtain

$$
\left\|u_{n+1}(t)-u_{n}(t)\right\|_{H} \leq\left\|A^{-\frac{1}{2}}\right\|_{H \rightarrow H}
$$

$\times \int_{m \omega}^{t}\left\|A^{1 / 2} s(t-y)\right\|_{H \rightarrow H}\left\|f\left(y, u_{n}(y), u_{y}(y-w), u(y-w)\right)-f\left(y, u_{n-1}(y), u_{y}(y-w), u(y-w)\right)\right\|_{H} d y$ $\leq \delta^{-\frac{1}{2}} L \int_{m \omega}^{t}\left\|u_{n}(y)-u_{n-1}(y)\right\|_{H} d y \leq \delta^{-\frac{1}{2}} L \int_{m \omega}^{t} \frac{\bar{M}\left(\delta^{-\frac{1}{2}} L(y-m \omega)\right)^{n}}{n !} d y=\frac{\bar{M}}{L} \frac{\left(\delta^{-\frac{1}{2}} L(t-m \omega)\right)^{n+1}}{(n+1) !}$

$$
\left\|u_{n+1}^{\prime}(t)-u_{n}^{\prime}(t)\right\|_{H}
$$

$\leq \int_{m \omega}^{t}\|c(t-y)\|_{H \rightarrow H}\left\|f\left(y, u_{n}(y), u_{y}(y-w), u(y-w)\right)-f\left(y, u_{n-1}(y), u_{y}(y-w), u(y-w)\right)\right\|_{H} d y$

$$
\leq \int_{m \omega}^{t} L\left\|u_{n}(y)-u_{n-1}(y)\right\|_{H} d s \leq \int_{m \omega}^{t} L \frac{\bar{M}}{L} \frac{\left(L \delta^{-\frac{1}{2}}(y-m \omega)\right)^{n}}{n !} d y=\frac{\bar{M}}{L \delta^{-\frac{1}{2}}} \frac{\left(L \delta^{-\frac{1}{2}}(t-m \omega)\right)^{n+1}}{(n+1) !} .
$$

\section{Therefore,}

$$
\left\|u_{n+1}(t)-u_{n}(t)\right\|_{H} \leq \frac{\bar{M}}{L} \frac{\left(\delta^{-\frac{1}{2}} L(t-m \omega)\right)^{n+1}}{(n+1) !},\left\|u_{n+1}^{\prime}(t)-u_{n}^{\prime}(t)\right\|_{H} \leq \frac{\bar{M}}{L \delta^{-\frac{1}{2}}} \frac{\left(L \delta^{-\frac{1}{2}}(t-m \omega)\right)^{n+1}}{(n+1) !}
$$


and

$$
\begin{gathered}
\left\|u_{n+1}(t)\right\|_{H} \leq \delta^{-\frac{1}{2}}\left[M_{m-1}+\widetilde{M}_{m-1}\right]+\delta^{-\frac{1}{2}} \bar{M}(t-m \omega)+\frac{\bar{M}}{L} \frac{\left(\delta^{-\frac{1}{2}} L(t-m \omega)\right)^{2}}{2 !} \cdots+\frac{\bar{M}}{L} \frac{\left(\delta^{-\frac{1}{2}} L(t-m \omega)\right)^{n+1}}{(n+1) !} \\
\left\|u_{n+1}^{\prime}(t)\right\|_{H} \leq M_{m-1}+\widetilde{M}_{m-1}+\bar{M}(t-m \omega)+\frac{\bar{M}}{L \delta^{-\frac{1}{2}}} \frac{\left(L \delta^{-\frac{1}{2}}(t-m \omega)\right)^{2}}{2 !}+\cdots+\frac{\bar{M}}{L \delta^{-\frac{1}{2}}} \frac{\left(L \delta^{-\frac{1}{2}}(t-m \omega)\right)^{n+1}}{(n+1) !}
\end{gathered}
$$

are true for any $n, n \geq 1$ by mathematical induction. From that and formulas (14) and (15) it follows that

$$
\begin{gathered}
\|u(t)\|_{H} \leq\left\|u_{0}(t)\right\|_{H} \\
+\sum_{i=0}^{\infty}\left\|u_{i+1}(t)-u_{i}(t)\right\|_{H} \leq \delta^{-\frac{1}{2}}[M+\tilde{M}]+\sum_{i=0}^{\infty} \frac{\bar{M}}{L} \frac{\left(\delta^{-\frac{1}{2}} L(t-m \omega)\right)^{i+1}}{(i+1) !} \\
\leq \delta^{-\frac{1}{2}}\left[M_{m-1}+\tilde{M}_{m-1}\right]+\frac{\bar{M}}{L} e^{\delta^{-\frac{1}{2}} L(t-m \omega)}, m \omega \leq t \leq(m+1) w \\
\left\|u^{\prime}(t)\right\|_{H} \leq\left\|u_{0}^{\prime}(t)\right\|_{H}+\sum_{i=0}^{\infty}\left\|u_{i+1}^{\prime}(t)-u_{i}^{\prime}(t)\right\|_{H} \\
\leq \delta^{-\frac{1}{2}}[M+\tilde{M}]+\sum_{i=0}^{\infty} \frac{\bar{M}}{L \delta^{-\frac{1}{2}}} \frac{\left(L \delta^{-\frac{1}{2}}(t-m \omega)\right)^{i+1}}{(i+1) !} \\
\leq M_{m-1}+\tilde{M}_{m-1}+\frac{\bar{M}}{L \delta^{-\frac{1}{2}}} e^{L \delta^{-\frac{1}{2}}(t-m \omega)}, m \omega \leq t \leq(m+1) w
\end{gathered}
$$

which proves the existence of a bounded solution of problem (1) in $[m \omega,(m+1) w] \times H$.

Now we will prove uniqueness of the bounded solution of the problem. Suppose that there is a bounded solution $v(t)$ of problem (1) and $v(t) \neq u(t)$. Denoting $z(t)=v(t)-u(t)$ and using Equation (1), we get

$$
\left\{\begin{array}{l}
\frac{d^{2} z(t)}{d t^{2}}+A z(t)=f\left(t, v(t), v_{t}(t-w), v(t-w)\right)-f\left(t, u(t), u_{t}(t-w), u(t-w)\right), \quad t>0 \\
z(t)=0,-w \leq t \leq 0
\end{array}\right.
$$

for $z(t)$.

Let $0 \leq t \leq w$. Since $v(t-w)=u(t-w)=\varphi(t-w)$, we can write

$$
\left\{\begin{array}{l}
\frac{d^{2} z(t)}{d t^{2}}+A z(t)=f\left(t, v(t), \varphi_{t}(t-w), \varphi(t-w)\right)-f\left(t, u(t), \varphi_{t}(t-w), \varphi(t-w)\right), \quad t>0 \\
z(t)=0,-w \leq t \leq 0
\end{array}\right.
$$

Therefore,

$$
z(t)=\int_{0}^{t} s(t-y)\left[f\left(y, v(y), \varphi_{y}(y-w), \varphi(y-w)\right)-f\left(y, u(y), \varphi_{y}(y-w), \varphi(y-w)\right)\right] d y .
$$

Applying estimates (3) and (6), we get

$$
\begin{gathered}
\|z(t)\|_{H} \leq\left\|A^{-\frac{1}{2}}\right\|_{H \rightarrow H} \\
\times \int_{0}^{t}\left\|A^{1 / 2} s(t-y)\right\|\left\|f\left(y, v(y), \varphi_{y}(y-w), \varphi(y-w)\right)-f\left(y, u(y), \varphi_{y}(y-w), \varphi(y-w)\right)\right\|_{H} d y \\
\leq L \delta^{-\frac{1}{2}} \int_{0}^{t}\|v(y)-u(y)\|_{H} d s \leq L \delta^{-\frac{1}{2}} \int_{0}^{t}\|z(y)\|_{H} d y .
\end{gathered}
$$


Applying the integral inequality, we get

$$
\|z(t)\|_{H} \leq 0
$$

From that it follows that $z(t)=0$ which proves the uniqueness of a bounded solution of problem (1) in $[0, w] \times H$. Using the same method and mathematical induction, we can establish the uniqueness of a bounded solution of problem (1) in $[0, \infty) \times H$. Theorem 1 is proved.

\section{Applications}

First, we consider the initial value problem for a semilinear hyperbolic equation with time delay and with nonlocal conditions

$$
\left\{\begin{array}{l}
u_{t t}(t, x)-\left(a(x) u_{x}(t, x)\right)_{x}+\delta u(t, x)=f\left(t, x, u(t, x), u_{t}(t-w, x), u(t-w, x)\right), \\
0<t<\infty, x \in(0, l) \\
u(t, x)=\varphi(t, x),-\omega \leq t \leq 0, x \in[0, l] \\
u(t, 0)=u(t, l), u_{x}(t, 0)=u_{x}(t, l), 0 \leq t<\infty,
\end{array}\right.
$$

where $a(x)$ and $\varphi(t, x)$ are given sufficiently smooth functions, $\delta>0$ is the sufficiently large number. Suppose that $a(x) \geq a>0$ and $a(l)=a(0)$.

Theorem 2. Suppose the following hypotheses:

1. For any $t,-w \leq t \leq 0$

$$
\|\varphi(t, .)\|_{W_{2}^{2}[0, l]} \leq M,\left\|\varphi^{\prime}\left(t_{,} .\right)\right\|_{W_{2}^{1}[0, l]} \leq \widetilde{M} .
$$

2. The function $f:[0, \infty) \times(0, l) \times L_{2}[0, l] \times L_{2}[0, l] \times L_{2}[0, l] \rightarrow L_{2}[0, l]$ be continuous and bounded, that is

$$
\|f(t, \cdot, u, v, w)\|_{L_{2}[0, l]} \leq \bar{M}
$$

and Lipschitz condition is satisfied uniformly with respect to $t, z, w$

$$
\|f(t, \cdot, u, z, w)-f(t, \cdot, v, z, w)\|_{L_{2}[0, l]} \leq L\|u-v\|_{L_{2}[0, l]} .
$$

3. Here and in future, $M, \widetilde{M}, \bar{M}, €$ are positive constants. Assume that all compatibility conditions are satisfied. Then there exists a unique solution to problem (17) which is bounded in $[0, \infty) \times L_{2}[0, l]$.

The proof of Theorem 2 is based on the abstract Theorem 1, on the self-adjointness and positivity in $L_{2}[0, l]$ of a differential operator $A$ defined by the formula

$$
A u=-\frac{d}{d x}\left(a(x) \frac{d u}{d x}\right)+\delta u
$$

with domain $D(A)=\left\{u \in W_{2}^{2}[0, l]: u(0)=u(l), u^{\prime}(0)=u^{\prime}(l)\right\}[51]$ and on estimates

$$
\|c\{t\}\|_{L_{2}[0, l] \rightarrow L_{2}[0, l]} \leq 1,\left\|(A)^{\frac{1}{2}} s\{t\}\right\|_{L_{2}[0, l] \rightarrow L_{2}[0, l]} \leq 1, \quad t \geq 0
$$


Second, we consider the initial value problem for a semilinear hyperbolic equation with time delay and with involution

$$
\left\{\begin{array}{l}
u_{t t}(t, x)-\left(a(x) u_{x}(t, x)\right)_{x}+\delta u(t, x)-\beta\left(a(-x) u_{x}(t,-x)\right)_{x} \\
=f\left(t, x, u(t, x), u_{t}(t-w, x), u(t-w, x)\right), 0<t<\infty, x \in(-l, l), \\
u(t, x)=\varphi(t, x),-\omega \leq t \leq 0, x \in[-l, l] \\
u(t,-l)=u(t, l)=0,0 \leq t<\infty
\end{array}\right.
$$

where $a(x)$ and $\varphi(t, x)$ are given sufficiently smooth functions, $\delta>0$ is the sufficiently large number. Suppose that $a \geq a(x)=a(-x) \geq \delta>0, \delta-a|\beta| \geq 0$.

Theorem 3. Suppose the following hypotheses:

1. For any $t,-w \leq t \leq 0$

$$
\|\varphi(t, .)\|_{W_{2}^{2}[-l, l]} \leq M,\left\|\varphi^{\prime}(t, .)\right\|_{W_{2}^{1}[-l, l]} \leq \tilde{M} .
$$

2. The function $f:[0, \infty) \times(-l, l) \times L_{2}[-l, l] \times L_{2}[-l, l] \times L_{2}[-l, l] \rightarrow L_{2}[-l, l]$ be continuous and bounded, that is

$$
\|f(t, \cdot, u, v, w)\|_{L_{2}[-l, l]} \leq \bar{M}
$$

and Lipschitz condition is satisfied uniformly with respect to $t, z, w$

$$
\|f(t, \cdot, u, z, w)-f(t, v, z, w)\|_{L_{2}[-l, l]} \leq L\|u-v\|_{L_{2}[-l, l]} .
$$

Assume that all compatibility conditions are satisfied. Then there exists a unique solution to problem (22) which is bounded in $[0, \infty) \times L_{2}[-l, l]$.

The proof of Theorem 3 is based on the abstract Theorem 1, on the self-adjointness and positivity in $L_{2}[-l, l]$ of a differential operator $A$ defined by the formula

$$
A v(x)=-\left(a(x) v_{x}(x)_{x}-\beta\left(a(-x) v_{x}(-x)\right)_{x}+\delta v(x)\right.
$$

with the domain $D(A)=\left\{u \in W_{2}^{2}[-l, l]: u(-l)=u(l)=0\right\}$ [52] and on the estimate

$$
\|c\{t\}\|_{L_{2}[-l, l] \rightarrow L_{2}[-l, l]} \leq 1,\left\|(A)^{\frac{1}{2}} s\{t\}\right\|_{L_{2}[-l, l] \rightarrow L_{2}[-l, l]} \leq 1, t \geq 0 .
$$

Third, let $\Omega \subset R^{m}$ be a bounded open domain with smooth boundary $S, \bar{\Omega}=\Omega \cup S$. In $[0, \infty) \times \Omega$ we consider the initial boundary value problem for a multidimensional semilinear delay differential equation of hyperbolic type

$$
\left\{\begin{array}{l}
\frac{\partial^{2} u(t, x)}{\partial t^{2}}-\sum_{r=1}^{m}\left(a_{r}(x) u_{x_{r}}\right) x_{r}+\delta u(t, x) \\
=f\left(t, x, u(t, x), u_{t}(t-w, x), u(t-w, x)\right), 0<t<\infty, x=\left(x_{1}, \ldots, x_{m}\right) \in \Omega, \\
u(t, x)=\varphi(t, x), \quad-\omega \leq t \leq 0, x \in \bar{\Omega}, \\
u(t, x)=0, \quad x \in S, \quad 0 \leq t<\infty,
\end{array}\right.
$$


where $a_{r}(x)$ and $\varphi(t, x)$ are given sufficiently smooth functions and $\delta>0$ is the sufficiently large number and $a_{r}(x)>0$.

Theorem 4. Suppose the following hypotheses:

1. For any $t,-w \leq t \leq 0$,

$$
\|\varphi(t, .)\|_{W_{2}^{2}(\bar{\Omega})} \leq M,\left\|\varphi^{\prime}(t, .)\right\|_{W_{2}^{1}(\bar{\Omega})} \leq \tilde{M} .
$$

2. The function $f:[0, \infty) \times Q \times L_{2}(\bar{\Omega}) \times L_{2}(\bar{\Omega}) \times L_{2}(\bar{\Omega}) \rightarrow L_{2}(\bar{\Omega})$ be continuous and bounded, that is

$$
\|f(t, u, v, w)\|_{L_{2}(\bar{\Omega})} \leq \bar{M}
$$

and Lipschitz condition is satisfied uniformly with respect to $t, z, w$

$$
\|f(t, u, z, w)-f(t, v, z, w)\|_{L_{2}(\bar{\Omega})} \leq L\|u-v\|_{L_{2}(\bar{\Omega})} .
$$

Assume that all compatibility conditions are satisfied. Then there exists a unique solution to problem (23) which is bounded in $[0, \infty) \times L_{2}(\bar{\Omega})$.

The proof of Theorem 4 is based on the abstract Theorem 1, on the self-adjointness and positivity in $L_{2}(\bar{\Omega})$ of a differential operator $A$ defined by the formula

$$
A u(x)=-\sum_{r=1}^{m}\left(a_{r}(x) u_{x_{r}}\right)_{x_{r}}+\delta u(x)
$$

with domain [53]

$$
D(A)=\left\{u(x): u(x), u_{x_{r}}(x),\left(a_{r}(x) u_{x_{r}}\right)_{x_{r}} \in L_{2}(\bar{\Omega}), 1 \leq r \leq m, u(x)=0, x \in S\right\}
$$

and on the estimate

$$
\|c\{t\}\|_{L_{2}(\bar{\Omega}) \rightarrow L_{2}(\bar{\Omega})} \leq 1,\left\|(A)^{\frac{1}{2}} s\{t\}\right\|_{L_{2}(\bar{\Omega}) \rightarrow L_{2}(\bar{\Omega})} \leq 1, t \geq 0
$$

and on the following theorem on the coercivity inequality for the solution of the elliptic differential problem in $L_{2}(\bar{\Omega})$.

Theorem 5. For the solutions of the elliptic differential problem

$$
\left\{\begin{array}{l}
A^{x} u(x)=\omega(x), \quad x \in \Omega, \\
u(x)=0, \quad x \in S,
\end{array}\right.
$$

the coercivity inequality [53]

$$
\sum_{r=1}^{m}\left\|u_{x_{r} x_{r}}\right\|_{L_{2}(\bar{\Omega})} \leq M_{1}\|\omega\|_{L_{2}(\bar{\Omega})} .
$$

is satisfied. Here $M_{1}$ is independent of $\omega(x)$. 
Fourth, in $[0, \infty) \times \Omega$ we consider the initial boundary value problem for a multidimensional semilinear delay hyperbolic equation

$$
\left\{\begin{array}{l}
\frac{\partial^{2} u(t, x)}{\partial t^{2}}-\sum_{r=1}^{m}\left(a_{r}(x) u_{x_{r}}\right) x_{r}+\delta u(t, x) \\
=f\left(t, x, u(t, x), u_{t}(t-w, x), u(t-w, x)\right), \quad x=\left(x_{1}, \ldots, x_{m}\right) \in \Omega, \\
u(t, x)=\varphi(t, x), \quad-\omega \leq t \leq 0, \quad x \in \bar{\Omega}, \\
\frac{\partial u}{\partial \vec{p}}(t, x)=0, \quad x \in S, \quad 0 \leq t<\infty,
\end{array}\right.
$$

where $a_{r}(x)$ and $\varphi(t, x)$ are given sufficiently smooth functions and $\delta>0$ is the sufficiently large number and $a_{r}(x)>0$. Here, $\vec{p}$ is the normal vector to $\Omega$.

Theorem 6. Suppose that assumptions of Theorem 4 hold. Assume that all compatibility conditions are satisfied. Then same stability estimates for the solution of (26) hold.

The proof of Theorem 6 is based on the abstract Theorem 3, on the self-adjointness and positivity of a differential operator $A$ defined by the formula

$$
A u(x)=-\sum_{r=1}^{m}\left(a_{r}(x) u_{x_{r}}\right)_{x_{r}}+\delta u(x)
$$

with domain

$$
D(A)=\left\{u(x): u(x), u_{x_{r}}(x),\left(a_{r}(x) u_{x_{r}}\right)_{x_{r}} \in L_{2}(\bar{\Omega}), 1 \leq r \leq m, \frac{\partial}{\partial \vec{p}} u(x)=0, x \in S\right\}
$$

in $L_{2}(\bar{\Omega})$ and on the following theorem on the coercivity inequality for the solution of the elliptic differential problem in $L_{2}(\bar{\Omega})$.

Theorem 7. For the solutions of the elliptic differential problem

$$
\left\{\begin{array}{l}
A^{x} u(x)=\omega(x), \quad x \in \Omega, \\
\frac{\partial u(x)}{\partial \vec{p}}=0, \quad x \in S
\end{array}\right.
$$

the coercivity inequality [53]

$$
\sum_{r=1}^{m}\left\|u_{x_{r} x_{r}}\right\|_{L_{2}(\bar{\Omega})} \leq M_{1}(\delta)\|\omega\|_{L_{2}(\bar{\Omega})}
$$

is satisfied. Here $M_{1}(\delta)$ is independent of $\omega(x)$. 


\section{The Main Theorem on Existence and Uniqueness of a Uniformly Bounded Solution of the Difference Scheme}

In the present section for the approximate solution of Equation (1) we will study the first order of accuracy difference scheme

$$
\begin{aligned}
& \frac{u^{k+1}-2 u^{k}+u^{k-1}}{\tau^{2}}+A u^{k+1}=f\left(t_{k}, u^{k}, \frac{u^{k-N}-u^{k-N-1}}{\tau}, u^{k-N}\right), \\
& t_{k}=k \tau, \quad 1 \leq k<\infty, \quad N \tau=\omega, \\
& \left(I+\tau^{2} A\right) \frac{u^{k+1}-u^{k}}{\tau}=\frac{u^{k}-u^{k-1}}{\tau}, \quad k=m N, \quad m=0,1, \ldots, \\
& u^{k}=\varphi_{k}, \quad \varphi_{k}=\varphi\left(t_{k}\right), \quad t_{k}=k \tau, \quad-N \leq k \leq 0 .
\end{aligned}
$$

The approach of proof of the theorem on the existence and uniqueness of a bounded solution of difference scheme (28) uniformly with respect to $\tau$ is based on reducing this difference scheme to an equivalent nonlinear equations. Equivalent nonlinear equations for the difference scheme (28) is

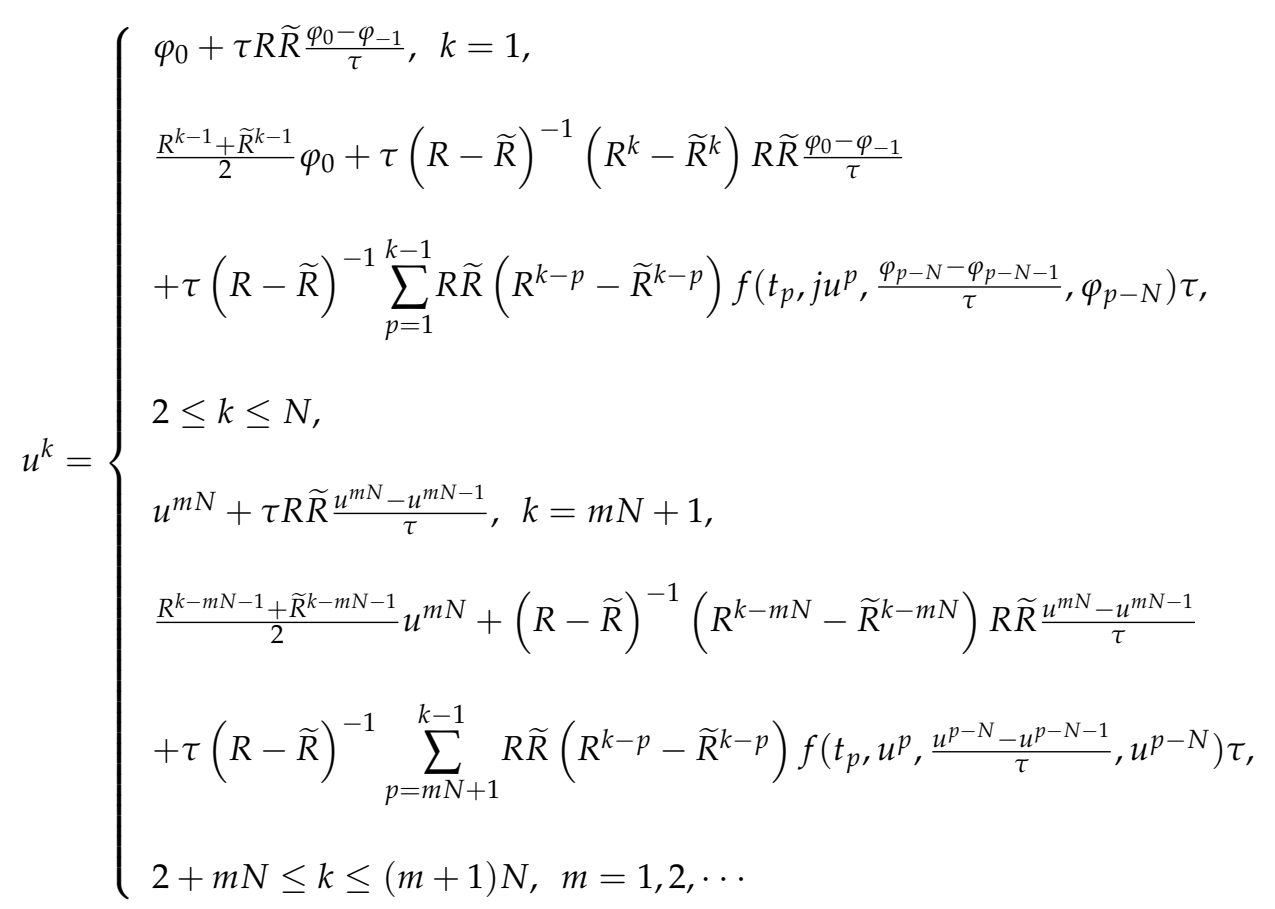

in $C_{\tau}(H)$ and the use of successive approximations. Here and in future $R=\left(I+\tau i A^{\frac{1}{2}}\right)^{-1}, \widetilde{R}=(I-$ $\left.\tau i A^{\frac{1}{2}}\right)^{-1}$ and $C_{\tau}(H)=C\left([0, \infty)_{\tau}, H\right)$ stands for the Banach space of the mesh functions $v^{\tau}=\left\{v^{l}\right\}_{l=0}^{\infty}$ defined on a grid space

$$
[0, \infty)_{\tau}=\left\{t_{k}=k \tau, k=0,1, \ldots, N \tau=w\right\}
$$

with values in $H$, equipped with the norm

$$
\left\|v^{\tau}\right\|_{C_{\tau}(H)}=\sup _{0 \leq l<\infty}\left\|v^{l}\right\|_{H}
$$


The recursive formula for the solution of difference scheme (28) is

$$
\left\{\begin{array}{l}
\frac{j u^{k+1}-2 j u^{k}+j u^{k-1}}{\tau^{2}}+A j u^{k+1}=f\left(t_{k},(j-1) u^{k}, \frac{u^{k-N}-u^{k-N-1}}{\tau}, u^{k-N}\right), \\
t_{k}=k \tau, m N+1 \leq k \leq(m+1) N, m=0,1,2, \cdots, N \tau=\omega, \\
\left(I+\tau^{2} A\right) \frac{j u^{k+1}-j u^{k}}{\tau}=\frac{j u^{k}-j u^{k-1}}{\tau}, k=m N, m=1, \ldots, \\
u^{k}=\varphi_{k}, \quad t_{k}=k \tau,-N \leq k \leq 0, \\
j=1,2, \cdots, 0 u^{k} \text { is given for any } k .
\end{array}\right.
$$

From Equations (29) and (30) it follows

$$
j u^{k}=\left\{\begin{array}{l}
\varphi_{0}+\tau R \widetilde{R} \frac{\varphi_{0}-\varphi_{-1}}{\tau}, k=1, \\
\frac{R^{k-1}+\widetilde{R}^{k-1}}{2} \varphi_{0}+\tau(R-\widetilde{R})^{-1}\left(R^{k}-\widetilde{R}^{k}\right) R \widetilde{R} \frac{\varphi_{0}-\varphi_{-1}}{\tau} \\
+\tau(R-\widetilde{R})^{-1} \sum_{p=1}^{k-1} R \widetilde{R}\left(R^{k-p}-\widetilde{R}^{k-p}\right) f\left(t_{p}, j u^{p}, \frac{\varphi_{p-N}-\varphi_{p-N-1}}{\tau}, \varphi_{p-N}\right) \tau, \\
2 \leq k \leq N, \quad k=m N+1, \\
u^{m N}+\tau R \widetilde{R} \frac{u^{m N}-u^{m N-1}}{\tau}, k \widetilde{R}^{k-m N-1}+\widetilde{R}^{k-m N-1} u^{m N}+\tau(R-\widetilde{R})^{-1}\left(R^{k-m N}-\widetilde{R}^{k-m N}\right) R \widetilde{R} \frac{u^{m N}-u^{m N-1}}{\tau} \\
+(R-\widetilde{R})^{-1} R \widetilde{R} \\
\quad \sum_{p=m N+1}^{k-1}\left(R^{k-p}-\widetilde{R}^{k-p}\right) f\left(t_{p},(j-1) u^{p}, \frac{u^{p-N}-u^{p-N-1}}{\tau}, u^{p-N}\right) \tau, \\
m N \leq k \leq(m+1) N, m=1,2, \cdots, j=1,2, \ldots,
\end{array}\right.
$$

where

$$
0 u^{k}=\left\{\begin{array}{l}
\varphi_{0}+R \widetilde{R} \frac{\varphi_{0}-\varphi_{-1}}{\tau}, k=1, \\
\frac{R^{k-1}+\widetilde{R}^{k-1}}{2} \varphi_{0}+\tau(R-\widetilde{R})^{-1}\left(R^{k}-\widetilde{R}^{k}\right) R \widetilde{R} \frac{\varphi_{0}-\varphi_{-1}}{\tau}, \\
2 \leq k \leq N, \\
u^{m N}+\tau R \widetilde{R} \frac{u^{m N}-u^{m N-1}}{\tau}, k=m N+1, \\
\frac{R^{k-m N-1}+\widetilde{R}^{k-m N-1}}{2} u^{m N}+\tau(R-\widetilde{R})^{-1}\left(R^{k-m N}-\widetilde{R}^{k-m N}\right) R \widetilde{R} \frac{u^{m N}-u^{m N-1}}{\tau} \\
2+m N \leq k \leq(m+1) N, m=1,2, \cdots
\end{array}\right.
$$


Let us give the lemma that will be needed below.

Lemma 2. The following estimates hold:

$$
\begin{gathered}
\|R\|_{H \rightarrow H} \leq 1,\|\tau A R\|_{H \rightarrow H} \leq 1,\|\widetilde{R}\|_{H \rightarrow H} \leq 1,\|\tau A \widetilde{R}\|_{H \rightarrow H} \leq 1, \\
\left\|\tau A\left(I+\tau^{2} A^{2}\right)^{-1}\right\|_{H \rightarrow H} \leq 1 .
\end{gathered}
$$

The proof of Lemma 2 is based on the spectral representation of the self-adjoint positive definite operator in a Hilbert space (see, [50]).

Theorem 8. Let the assumptions of Theorem 1 be satisfied. Then, there exists a unique solution $u^{\tau}=\left\{u^{k}\right\}_{k=0}^{\infty}$ of difference scheme (28) which is bounded in $C_{\tau}(H)$ of uniformly with respect to $\tau$.

Proof. Step 1. Uniformly boundedness of solution of difference scheme (28) on $[0, w]_{\tau}$. Assume that $1 \leq k \leq N$. According to the method of recursive approximation (31), we get

$$
u^{k}=0 u^{k}+\sum_{i=0}^{\infty}\left[(i+1) u^{k}-i u^{k}\right]
$$

where

$$
\begin{gathered}
0 u^{1}=\varphi_{0}+\tau R \widetilde{R} \frac{\varphi_{0}-\varphi_{-1}}{\tau}, \\
0 u^{k}=\frac{R^{k-1}+\widetilde{R}^{k-1}}{2} \varphi_{0}+\tau(R-\widetilde{R})^{-1}\left(R^{k}-\widetilde{R}^{k}\right) R \widetilde{R} \frac{\varphi_{0}-\varphi_{-1}}{\tau}, 2 \leq k \leq N, \\
j u^{1}=\varphi_{0}+\tau R \widetilde{R} \frac{\varphi_{0}-\varphi_{-1}}{\tau}, j=1,2, \cdots, \\
j u^{k}=\frac{R^{k-1}+\widetilde{R}^{k-1}}{2} \varphi_{0}+\tau(R-\widetilde{R})^{-1}\left(R^{k}-\widetilde{R}^{k}\right) R \widetilde{R} \frac{\varphi_{0}-\varphi_{-1}}{\tau}+\tau(R-\widetilde{R})^{-1} \\
\times \sum_{p=1}^{k-1} R \widetilde{R}\left(R^{k-p}-\widetilde{R}^{k-p}\right) f\left(t_{p}, j u^{p}, \frac{\varphi_{p-N}-\varphi_{p-N-1}}{\tau}, \varphi_{p-N}\right) \tau, 2 \leq k \leq N, j=1,2, \cdots
\end{gathered}
$$

Applying formula (35), the triangle inequality and estimates (5), (32), and (33), we get

$$
\begin{aligned}
& \left\|0 u^{1}\right\|_{H} \leq\left\|A^{-1}\right\|_{H \rightarrow H}\left[\left\|A \varphi_{0}\right\|_{H}+\left\|\tau A^{\frac{1}{2}} R \widetilde{R}\right\|_{H \rightarrow H}\left\|A^{\frac{1}{2}} \frac{\varphi_{0}-\varphi_{-1}}{\tau}\right\|_{H}\right] \leq \delta^{-1}[M+\widetilde{M}], \\
& \left\|\frac{0 u^{1}-\varphi_{0}}{\tau}\right\|_{H} \leq\left\|A^{-\frac{1}{2}}\right\|_{H \rightarrow H}\|R \widetilde{R}\|_{H \rightarrow H}\left\|A^{\frac{1}{2}} \frac{\varphi_{0}-\varphi_{-1}}{\tau}\right\|_{H} \leq \delta^{-\frac{1}{2}} \widetilde{M}, \\
& \left\|0 u^{k}\right\|_{H} \leq\left\|A^{-1}\right\|_{H \rightarrow H}\left[\frac{1}{2}\left[\|R\|_{H \rightarrow H}^{k-1}+\|\widetilde{R}\|_{H \rightarrow H}^{k-1}\right]\left\|A \varphi_{0}\right\|_{H}+\frac{1}{2}\left[\|R\|_{H \rightarrow H}^{k}+\|\widetilde{R}\|_{H \rightarrow H}^{k}\right]\right. \\
& \left.\times\left\|A^{\frac{1}{2}} \frac{\varphi_{0}-\varphi_{-1}}{\tau}\right\|_{H}\right] \leq \delta^{-1}\left[\left\|A \varphi_{0}\right\|_{H}+\left\|A^{\frac{1}{2}} \frac{\varphi_{0}-\varphi_{-1}}{\tau}\right\|_{H}\right] \leq \delta^{-1}[M+\widetilde{M}], \\
& \left\|\frac{0 u^{k}-0 u^{k-1}}{\tau}\right\|_{H} \leq\left\|A^{-\frac{1}{2}}\right\|_{H \rightarrow H}\left[\frac{1}{2}\left[\|R\|_{H \rightarrow H}^{k-1}+\|\widetilde{R}\|_{H \rightarrow H}^{k-1}\right]\left\|A \varphi_{0}\right\|_{H}+\frac{1}{2}\left[\|R\|_{H \rightarrow H}^{k}+\|\widetilde{R}\|_{H \rightarrow H}^{k}\right]\right. \\
& \left.\times\left\|A^{\frac{1}{2}} \frac{\varphi_{0}-\varphi_{-1}}{\tau}\right\|_{H}\right] \leq \delta^{-\frac{1}{2}}\left[\left\|A \varphi_{0}\right\|_{H}+\left\|A^{\frac{1}{2}} \frac{\varphi_{0}-\varphi_{-1}}{\tau}\right\|_{H}\right] \leq \delta^{-\frac{1}{2}}[M+\tilde{M}]
\end{aligned}
$$


for any $2 \leq k \leq N$. Applying formula (36), and estimates (6), (32), and (33), we get

$$
\begin{gathered}
\left\|1 u^{1}-0 u^{1}\right\|_{H}=0, \\
\left\|1 u^{k}-0 u^{k}\right\|_{H} \leq\left\|A^{-\frac{1}{2}}\right\|_{H \rightarrow H} \\
\times \sum_{p=1}^{k-1} \frac{1}{2}\left[\|R\|_{H \rightarrow H}^{k-p}+\|\widetilde{R}\|_{H \rightarrow H}^{k-p}\right]\left\|f\left(t_{p}, 1 u^{p}, \frac{\varphi_{p-N}-\varphi_{p-N-1}}{\tau}, \varphi_{p-N}\right)\right\|_{H} \tau \leq \delta^{-\frac{1}{2}} \bar{M} t_{k} \\
\left\|\frac{1 u^{1}-\varphi_{0}}{\tau}-\frac{0 u^{1}-\varphi_{0}}{\tau}\right\|_{H}=0, \\
\left\|\frac{1 u^{k}-1 u^{k-1}}{\tau}-\frac{0 u^{k}-0 u^{k-1}}{\tau}\right\|_{H} \\
\leq \sum_{p=1}^{k-1} \frac{1}{2}\left[\|R\|_{H \rightarrow H}^{k-p}+\|\widetilde{R}\|_{H \rightarrow H}^{k-p}\right]\left\|f\left(t_{p}, 1 u^{p}, \frac{\varphi_{p-N}-\varphi_{p-N-1}}{\tau}, \varphi_{p-N}\right)\right\|_{H} \tau \leq \bar{M} t_{k}
\end{gathered}
$$

for any $k=2, \cdots, N$. Using the triangle inequality, we get

$$
\begin{gathered}
\left\|1 u^{k}\right\|_{H} \leq \delta^{-1}[M+\tilde{M}]+\delta^{-\frac{1}{2}} \bar{M} t_{k} \\
\left\|\frac{1 u^{k}-1 u^{k-1}}{\tau}\right\|_{H} \leq \delta^{-\frac{1}{2}}[M+\tilde{M}]+\bar{M} t_{k}
\end{gathered}
$$

for any $k=1, \cdots, N$. Applying formula (36), and estimates (7), (32), and (33), we get

$$
\begin{gathered}
\left\|2 u^{1}-1 u^{1}\right\|_{H}=0, \\
\left\|2 u^{k}-1 u^{k}\right\|_{H} \leq\left\|A^{-\frac{1}{2}}\right\|_{H \rightarrow H} \sum_{p=1}^{k-1} \frac{1}{2}\left[\|R\|_{H \rightarrow H}^{k-p}+\|\widetilde{R}\|_{H \rightarrow H}^{k-p}\right] \\
\times\left\|f\left(t_{p}, 1 u^{p}, \frac{\varphi_{p-N}-\varphi_{p-N-1}}{\tau}, \varphi_{p-N}\right)-f\left(t_{p}, 0 u^{p}, \frac{\varphi_{p-N}-\varphi_{p-N-1}}{\tau}, \varphi_{p-N}\right)\right\|_{H} \tau \\
\leq \delta^{-\frac{1}{2}} \sum_{p=1}^{k-1} L\left\|1 u^{p}-0 u^{p}\right\|_{H} \tau \leq \delta^{-\frac{1}{2}} \sum_{p=1}^{k-1} L \delta^{-\frac{1}{2}} \bar{M} t_{p} \tau \leq \delta^{-1} L \bar{M} \frac{t_{k}^{2}}{2 !}, \\
\left\|\frac{2 u^{1}-2 u^{0}}{\tau}-\frac{1 u^{1}-1 u^{0}}{\tau}\right\|_{H}=0, \\
\times\left\|f\left(t_{p}, 1 u^{p}, \frac{\varphi_{p-N}-\varphi_{p-N-1}}{\tau}, \varphi_{p-N}\right)-f\left(t_{p}, 0 u^{p}, \frac{\varphi_{p-N}-\varphi_{p-N-1}}{\tau}, \varphi_{p-N}\right)\right\|_{H} \tau \\
\left.\leq \sum_{p=1}^{k-1} L\left\|1 u^{k}-0 u^{p}\right\|_{H} \tau \leq \sum_{p=1}^{k-1} L \delta^{-\frac{1}{2}} \bar{M} t_{p} \tau \leq \frac{\bar{M}}{L \delta^{-\frac{1}{2}}} \frac{\left(\delta^{-\frac{1}{2}} L t_{k}\right)^{2}}{2 !},\|R\|_{H \rightarrow H}^{k-p}+\|\widetilde{R}\|_{H \rightarrow H}^{k-p}\right]
\end{gathered}
$$

for any $k=2, \cdots, N$. Using the triangle inequality, we get

$$
\left\|2 u^{k}\right\|_{H} \leq \delta^{-1}[M+\tilde{M}]+\delta^{-\frac{1}{2}} \bar{M} t_{k}+\frac{\bar{M}}{L} \frac{\left(\delta^{-\frac{1}{2}} L t_{k}\right)^{2}}{2 !},
$$




$$
\left\|\frac{2 u^{k}-2 u^{k-1}}{\tau}\right\|_{H} \leq \delta^{-\frac{1}{2}}[M+\tilde{M}]+\bar{M} t_{k}+\frac{\bar{M}}{L \delta^{-\frac{1}{2}}} \frac{\left(\delta^{-\frac{1}{2}} L t_{k}\right)^{2}}{2 !}
$$

for any $k=1, \cdots, N$. Let

$$
\begin{gathered}
\left\|n u^{k}-(n-1) u^{k}\right\|_{H} \leq \frac{\bar{M}}{L} \frac{\left(L \delta^{-\frac{1}{2}} t_{k}\right)^{n}}{n !}, \\
\left\|\frac{n u^{k}-n u^{k-1}}{\tau}-\frac{(n-1) u^{k}-(n-1) u^{k-1}}{\tau}\right\|_{H} \leq \frac{\bar{M}}{L \delta^{-\frac{1}{2}}} \frac{\left(L \delta^{-\frac{1}{2}} t_{k}\right)^{n}}{n !}, \\
\left\|n u^{k}\right\|_{H} \leq \delta^{-1}[M+\tilde{M}]+\delta^{-\frac{1}{2}} \bar{M} t_{k}+\bar{M} \frac{\left(\delta^{-\frac{1}{2}} L t_{k}\right)^{2}}{2 !}+\cdots+\frac{\bar{M}}{L} \frac{\left(L \delta^{-\frac{1}{2}} t_{k}\right)^{n}}{n !}, \\
\left\|\frac{n u^{k}-n u^{k-1}}{\tau}\right\|_{H} \leq \delta^{-\frac{1}{2}}[M+\tilde{M}]+\bar{M} t_{k} \\
+\frac{\bar{M}}{L \delta^{-\frac{1}{2}}} \frac{\left(\delta^{-\frac{1}{2}} L t_{k}\right)^{2}}{2 !}+\cdots+\frac{\bar{M}}{L \delta^{-\frac{1}{2}}} \frac{\left(L \delta^{-\frac{1}{2}} t_{k}\right)^{n}}{n !}
\end{gathered}
$$

for any $k=1, \cdots, N$. Applying formula (36), and estimates (7), (32), and (33), we get

$$
\begin{gathered}
\left\|(n+1) u^{1}-n u^{1}\right\|_{H}=0, \\
\left\|(n+1) u^{k}-n u^{k}\right\|_{H} \leq\left\|A^{-\frac{1}{2}}\right\|_{H \rightarrow H} \sum_{p=1}^{k-1} \frac{1}{2}\left[\|R\|_{H \rightarrow H}^{k-p}+\|\widetilde{R}\|_{H \rightarrow H}^{k-p}\right] \\
\times\left\|f\left(t_{p}, n u^{p}, \frac{\varphi_{p-N}-\varphi_{p-N-1}}{\tau}, \varphi_{p-N}\right)-f\left(t_{p},(n-1) u^{p}, \frac{\varphi_{p-N}-\varphi_{p-N-1}}{\tau}, \varphi_{p-N}\right)\right\|_{H} \tau \\
\leq \delta^{-\frac{1}{2}} \sum_{p=1}^{k-1} L\left\|n u^{p}-(n-1) u^{p}\right\|_{H} \tau \leq \delta^{-\frac{1}{2}} L \sum_{p=1}^{k-1} \frac{\bar{M}}{L} \frac{\left(L \delta^{-\frac{1}{2}} t_{p}\right)^{n}}{n !} \tau \leq \frac{\bar{M}}{L} \frac{\left(L \delta^{-\frac{1}{2}} t_{k}\right)^{n+1}}{(n+1) !}, \\
\left\|\frac{(n+1) u^{1}-n u^{0}}{\tau}-\frac{n u^{1}-n u^{0}}{\tau}\right\|_{H}=0, \\
\left\|f\left(t_{p}, n u^{p}, \frac{\varphi_{p-N}-\varphi_{p-N-1}}{\tau}, \varphi_{p-N}\right)-f\left(t_{p},(n-1) u^{p}, \frac{\varphi_{p-N}-\varphi_{p-N-1}}{\tau}, \varphi_{p-N}\right)\right\|_{H} \tau \\
\leq \sum_{p=1}^{k-1} L\left\|n u^{p}-(n-1) u^{p}\right\|_{H} \tau \leq \sum_{p=1}^{k-1} L \frac{\bar{M}}{L \delta^{-\frac{1}{2}}} \frac{\left(L \delta^{-\frac{1}{2}} t_{p}\right)^{n}}{n !} \tau \leq \frac{\bar{M}}{L \delta^{-\frac{1}{2}}} \frac{\left(L \delta^{-\frac{1}{2}} t_{k}\right)^{n+1}}{(n+1) !}
\end{gathered}
$$

for any $k=2, \cdots, N$. Using the triangle inequality, we get

$$
\begin{gathered}
\left\|(n+1) u^{k}\right\|_{H} \leq \delta^{-1}[M+\tilde{M}]+\delta^{-\frac{1}{2}} \bar{M} t_{k}+\frac{\bar{M}}{L} \frac{\left(\delta^{-\frac{1}{2}} L t_{k}\right)^{2}}{2 !}+\cdots+\frac{\bar{M}}{L} \frac{\left(L \delta^{-\frac{1}{2}} t_{k}\right)^{n+1}}{(n+1) !} \\
\left\|\frac{(n+1) u^{k}-(n+1) u^{k-1}}{\tau}\right\|_{H} \leq \delta^{-\frac{1}{2}}[M+\tilde{M}]+\bar{M} t_{k}
\end{gathered}
$$




$$
+\frac{\bar{M}}{L \delta^{-\frac{1}{2}}} \frac{\left(\delta^{-\frac{1}{2}} L t_{k}\right)^{2}}{2 !}+\cdots+\frac{\bar{M}}{L \delta^{-\frac{1}{2}}} \frac{\left(L \delta^{-\frac{1}{2}} t_{k}\right)^{n+1}}{(n+1) !}
$$

for any $k=1, \cdots, N$. Therefore, for any $n, n \geq 1$, we have that

$$
\begin{gathered}
\left\|(n+1) u^{k}-n u^{k}\right\|_{H} \leq \frac{\bar{M}}{L} \frac{\left(L \delta^{-\frac{1}{2}} t_{k}\right)^{n+1}}{(n+1) !} \\
\left\|\frac{(n+1) u^{k}-(n+1) u^{k-1}}{\tau}-\frac{n u^{k}-n u^{k-1}}{\tau}\right\|_{H} \leq \frac{\bar{M}}{L \delta^{-\frac{1}{2}}} \frac{\left(L \delta^{-\frac{1}{2}} t_{k}\right)^{n+1}}{(n+1) !}
\end{gathered}
$$

and

$$
\begin{gathered}
\left\|(n+1) u^{k}\right\|_{H} \leq \delta^{-1}[M+\tilde{M}]+\delta^{-\frac{1}{2}} \bar{M} t_{k}+\frac{\bar{M}}{L} \frac{\left(\delta^{-\frac{1}{2}} L t_{k}\right)^{2}}{2 !}+\cdots+\frac{\bar{M}}{L} \frac{\left(L \delta^{-\frac{1}{2}} t_{k}\right)^{n+1}}{(n+1) !}, \\
\left\|\frac{(n+1) u^{k}-(n+1) u^{k-1}}{\tau}\right\|_{H} \leq \delta^{-\frac{1}{2}}[M+\tilde{M}]+\bar{M} t_{k} \\
+\frac{\bar{M}}{L \delta^{-\frac{1}{2}}} \frac{\left(\delta^{-\frac{1}{2}} L t_{k}\right)^{2}}{2 !}+\cdots+\frac{\bar{M}}{L \delta^{-\frac{1}{2}}} \frac{\left(L \delta^{-\frac{1}{2}} t_{k}\right)^{n+1}}{(n+1) !}
\end{gathered}
$$

for any $k=1, \cdots, N$ by mathematical induction. In a similar manner, for any $n$, we can obtain

$$
\left\|A^{\frac{1}{2}}(n+1) u^{k}-A^{\frac{1}{2}} n u^{k}\right\|_{H} \leq \frac{\bar{M}}{L \delta^{-\frac{1}{2}}} \frac{\left(L \delta^{-\frac{1}{2}} t_{k}\right)^{n+1}}{(n+1) !}
$$

and

$$
\left\|(n+1) u^{k}\right\|_{H} \leq \delta^{-\frac{1}{2}}[M+\tilde{M}]+\bar{M} t_{k}+\frac{\bar{M}}{L \delta^{-\frac{1}{2}}} \frac{\left(L \delta^{-\frac{1}{2}} t\right)^{2}}{2 !}+\cdots+\frac{\bar{M}}{L \delta^{-\frac{1}{2}}} \frac{\left(L \delta^{-\frac{1}{2}} t_{k}\right)^{n+1}}{(n+1) !} .
$$

From that and formula (34) it follows that

$$
\begin{aligned}
& \left\|u^{k}\right\|_{H} \leq\left\|0 u^{k}\right\|_{H}+\sum_{i=0}^{\infty}\left\|(i+1) u^{k}-i u^{k}\right\|_{H} \\
& \leq \delta^{-1}[M+\tilde{M}]+\sum_{i=0}^{\infty} \frac{\bar{M}}{L} \frac{\left(\delta^{-\frac{1}{2}} L t_{k}\right)^{i+1}}{(i+1) !} \leq \delta^{-1}[M+\tilde{M}]+\frac{\bar{M}}{L} e^{\delta^{-\frac{1}{2}} L t_{k}}, 1 \leq k \leq N, \\
& \left\|\frac{u^{k}-u^{k-1}}{\tau}\right\|_{H} \leq\left\|\frac{0 u^{k}-0 u^{k-1}}{\tau}\right\|_{H}+\sum_{i=0}^{\infty}\left\|\frac{(i+1) u^{k}-(i+1) u^{k-1}}{\tau}-\frac{i u^{k}-i u^{k-1}}{\tau}\right\|_{H} \\
& \leq \delta^{-\frac{1}{2}}[M+\tilde{M}]+\sum_{i=0}^{\infty} \frac{\bar{M}}{L \delta^{-\frac{1}{2}}} \frac{\left(L \delta^{-\frac{1}{2}} t_{k}\right)^{i+1}}{(i+1) !} \leq \delta^{-\frac{1}{2}}[M+\tilde{M}]+\frac{\bar{M}}{L \delta^{-\frac{1}{2}}} e^{\delta^{-\frac{1}{2}} L t_{k}}, \quad 1 \leq k \leq N, \\
& \left\|A^{\frac{1}{2}} u^{k}\right\|_{H} \leq\left\|A^{\frac{1}{2}} 0 u^{k}\right\|_{H}+\sum_{i=0}^{\infty}\left\|A^{\frac{1}{2}}(i+1) u^{k}-A^{\frac{1}{2}} i u^{k}\right\|_{H} \\
& \leq \delta^{-\frac{1}{2}}[M+\tilde{M}]+\sum_{i=0}^{\infty} \frac{\bar{M}}{L \delta^{-\frac{1}{2}}} \frac{\left(L \delta^{-\frac{1}{2}} t_{k}\right)^{i+1}}{(i+1) !} \leq \delta^{-\frac{1}{2}}[M+\tilde{M}]+\frac{\bar{M}}{L \delta^{-\frac{1}{2}}} e^{\delta^{-\frac{1}{2}} L t_{k}}, \quad 1 \leq k \leq N
\end{aligned}
$$

which proves the existence of a bounded solution of difference scheme (28) in $[0, w]_{\tau} \times H$ of uniformly with respect to $\tau$. 
Step 2. Uniformly boundedness of solution of difference scheme (28) on $[m w,(m+1) w]_{\tau}$. We consider solution of difference scheme (28) in $m N \leq k \leq(m+1) N, m=1,2, \ldots$. Then, according to the method of recursive approximation (31), we get

$$
u^{k}=0 u^{k}+\sum_{i=0}^{\infty}\left[(i+1) u^{k}-i u^{k}\right]
$$

where

$$
\begin{gathered}
0 u^{m N+1}=u^{m N}+\tau R \widetilde{R} \frac{u^{m N}-u^{m N-1}}{\tau}, \\
0 u^{k}=\frac{R^{k-m N-1}+\widetilde{R}^{k-m N-1}}{2} u^{m N} \\
+\tau(R-\widetilde{R})^{-1}\left(R^{k-m N}-\widetilde{R}^{k-m N}\right) R \widetilde{R} \frac{u^{m N}-u^{m N-1}}{\tau}, \\
2+m N \leq k \leq(m+1) N, m=1,2, \cdots, \\
j u^{n N+1}=u^{m N}+\tau R \widetilde{R} \frac{u^{m N}-u^{m N-1}}{\tau}, j=1,2, \cdots, \\
j u^{k}=\frac{R^{k-m N-1}+\widetilde{R}^{k-m N-1}}{2} u^{m N}+\tau(R-\widetilde{R})^{-1}\left(R^{k-m N}-\widetilde{R}^{k-m N}\right) R \widetilde{R} \frac{u^{m N}-u^{m N-1}}{\tau} \\
+\tau(R-\widetilde{R})^{-1} \sum_{p=m N+1}^{k-1} R \widetilde{R}\left(R^{k-p}-\widetilde{R}^{k-p}\right) f\left(t_{p},(j-1) u^{p}, \frac{u^{p-N}-u^{p-N-1}}{\tau}, u^{p-N}\right) \tau, \\
2+m N \leq k \leq(m+1) N, m=1,2, \cdots, j=1,2, \cdots .
\end{gathered}
$$

Assume that difference scheme (28) in $[(m-1) N, m N]_{\tau} \times H$ of uniformly with respect to $\tau$ and

$$
\left\|A^{1 / 2} u^{k}\right\|_{H} \leq M_{m-1},\left\|\frac{u^{k}-u^{k-1}}{\tau}\right\|_{H} \leq \widetilde{M}_{m-1} .
$$

Applying formula (38), the triangle inequality and estimates (32), (33), and (40), we get

$$
\begin{aligned}
& \left\|0 u^{m N+1}\right\|_{H} \leq\left\|A^{-\frac{1}{2}}\right\|_{H \rightarrow H}\left[\left\|A^{\frac{1}{2}} u^{m N}\right\|_{H}+\left\|\tau A^{\frac{1}{2}} R \widetilde{R}\right\|_{H \rightarrow H}\left\|\frac{u^{m N}-u^{m N-1}}{\tau}\right\|_{H}\right] \leq \delta^{-\frac{1}{2}}\left[M_{m-1}+\widetilde{M}_{m-1}\right], \\
& \left\|\frac{0 u^{m N+1}-u^{m N}}{\tau}\right\|_{H} \leq\|R \widetilde{R}\|_{H \rightarrow H}\left\|\frac{u^{m N}-u^{m N-1}}{\tau}\right\|_{H} \leq \widetilde{M}_{m-1}, \\
& \left\|0 u^{k}\right\|_{H} \leq\left\|A^{-\frac{1}{2}}\right\|_{H \rightarrow H}\left[\frac{1}{2}\left[\|R\|_{H \rightarrow H}^{k-1}+\|\widetilde{R}\|_{H \rightarrow H}^{k-1}\right]\left\|A^{\frac{1}{2}} u^{m N}\right\|_{H}+\frac{1}{2}\left[\|R\|_{H \rightarrow H}^{k}+\|\widetilde{R}\|_{H \rightarrow H}^{k}\right]\right. \\
& \left.\times\left\|\frac{u^{m N}-u^{m N-1}}{\tau}\right\|_{H}\right] \leq \delta^{-\frac{1}{2}}\left[\left\|A^{\frac{1}{2}} u^{m N}\right\|_{H}+\left\|\frac{u^{m N}-u^{m N-1}}{\tau}\right\|_{H}\right] \leq \delta^{-\frac{1}{2}}\left[M_{m-1}+\widetilde{M}_{m-1}\right], \\
& \left\|\frac{0 u^{k}-0 u^{k-1}}{\tau}\right\|_{H} \leq\left[\frac{1}{2}\left[\|R\|_{H \rightarrow H}^{k-1}+\|\widetilde{R}\|_{H \rightarrow H}^{k-1}\right]\left\|A^{\frac{1}{2}} u^{m N}\right\|_{H}+\frac{1}{2}\left[\|R\|_{H \rightarrow H}^{k}+\|\widetilde{R}\|_{H \rightarrow H}^{k}\right]\right. \\
& \left.\times\left\|\frac{u^{m N}-u^{m N-1}}{\tau}\right\|_{H}\right] \leq\left\|A u^{m N}\right\|_{H}+\left\|A^{\frac{1}{2}} \frac{u^{m N}-u^{m N-1}}{\tau}\right\|_{H} \leq M_{m-1}+\tilde{M}_{m-1}
\end{aligned}
$$

for any $2+m N \leq k \leq(m+1) N$. Applying formula (39), and estimates (6), (32) and (33), we get

$$
\begin{gathered}
\left\|1 u^{m N+1}-0 u^{m N+1}\right\|_{H}=0, \\
\left\|1 u^{k}-0 u^{k}\right\|_{H} \leq\left\|A^{-\frac{1}{2}}\right\|_{H \rightarrow H} \sum_{p=m N+1}^{k-1} \frac{1}{2}\left[\|R\|_{H \rightarrow H}^{k-p}+\|\widetilde{R}\|_{H \rightarrow H}^{k-p}\right] \\
\times\left\|f\left(t_{p},(j-1) u^{p}, \frac{u^{p-N}-u^{p-N-1}}{\tau}, u^{p-N}\right)\right\|_{H} \tau \leq \delta^{-\frac{1}{2}} \bar{M}\left(t_{k}-m N\right),
\end{gathered}
$$




$$
\begin{gathered}
\left\|\frac{1 u^{m N+1}-u^{m N}}{\tau}-\frac{0 u^{m N+1}-u^{m N}}{\tau}\right\|_{H}=0, \\
\left\|\frac{1 u^{k}-1 u^{k-1}}{\tau}-\frac{0 u^{k}-0 u^{k-1}}{\tau}\right\|_{H} \\
\leq \sum_{p=m N+1}^{k-1} \frac{1}{2}\left[\|R\|_{H \rightarrow H}^{k-p}+\|\widetilde{R}\|_{H \rightarrow H}^{k-p}\right]\left\|f\left(t_{p}, 1 u^{p}, \frac{u^{p-N}-u^{p-N-1}}{\tau}, u^{p-N}\right)\right\|_{H} \tau \leq \bar{M}\left(t_{k}-m N\right)
\end{gathered}
$$

for any $2+m N \leq k \leq(m+1) N$. Using the triangle inequality, we get

$$
\begin{gathered}
\left\|1 u^{k}\right\|_{H} \leq \delta^{-\frac{1}{2}}[M+\tilde{M}]+\delta^{-\frac{1}{2}} \bar{M}\left(t_{k}-m N\right), \\
\left\|\frac{1 u^{k}-1 u^{k-1}}{\tau}\right\|_{H} \leq M_{m-1}+\widetilde{M}_{m-1}+\bar{M}\left(t_{k}-m N\right)
\end{gathered}
$$

for any $1+m N \leq k \leq(m+1) N$. Applying formula (39), and estimates (7), (32), and (33), we get

$$
\begin{gathered}
\left\|2 u^{1}-1 u^{1}\right\|_{H}=0, \\
\left\|2 u^{k}-1 u^{k}\right\|_{H} \leq\left\|A^{-\frac{1}{2}}\right\|_{H \rightarrow H} \sum_{p=m N+1}^{k-1} \frac{1}{2}\left[\|R\|_{H \rightarrow H}^{k-p}+\|\widetilde{R}\|_{H \rightarrow H}^{k-p}\right] \\
\times\left\|f\left(t_{p}, 1 u^{p}, \frac{u^{p-N}-u^{p-N-1}}{\tau}, u^{p-N}\right)-f\left(t_{p}, 0 u^{p}, \frac{u^{p-N}-u^{p-N-1}}{\tau}, u^{p-N}\right)\right\|_{H} \tau \\
\leq \delta^{-\frac{1}{2}} \sum_{p=m N+1}^{k-1} L\left\|1 u^{p}-0 u^{p}\right\|_{H} \tau \leq \delta^{-\frac{1}{2}} \sum_{p=m N+1}^{k-1} L \delta^{-\frac{1}{2}} \bar{M} t_{p-m N} \tau \leq \delta^{-1} L \bar{M} \frac{\left(t_{k}-m N\right)^{2}}{2 !}, \\
\quad\left\|\frac{2 u^{1}-2 u^{0}}{\tau}-\frac{1 u^{1}-1 u^{0}}{\tau}\right\|_{H}=0, \\
\times\left\|_{\|^{k}-2 u^{k-1}}^{\tau}-\frac{1 u^{k}-1 u^{k-1}}{\tau}\right\|_{H} \leq \sum_{p=m N+1}^{k-1} \frac{1}{2}\left[\|R\|_{H \rightarrow H}^{k-p}+\|\widetilde{R}\|_{H \rightarrow H}^{k-p}\right] \\
\left.\leq \sum_{p=m N+1}^{k-1} L\left\|1 u^{p}-0 u^{p}\right\|_{H} \tau \leq \sum_{p=m N+1}^{k-1} L \delta^{-\frac{1}{2}} \bar{M} t_{p-m N} \tau \leq \frac{\bar{M}}{L \delta^{-\frac{1}{2}}} \frac{\left(\delta^{-\frac{1}{2}} L\left(t_{k}-m N\right)\right)^{2}}{2 !}, u^{p-N}\right)-f\left(t_{p}, 0 u^{p}, \frac{u^{p-N}-u^{p-N-1}}{\tau}, u^{p-N}\right) \|_{H} \tau
\end{gathered}
$$

for any $2+m N \leq k \leq(m+1) N$. Using the triangle inequality, we get

$$
\begin{gathered}
\left\|2 u^{k}\right\|_{H} \leq \delta^{-\frac{1}{2}}[M+\tilde{M}]+\delta^{-\frac{1}{2}} \bar{M}\left(t_{k}-m N\right)+\frac{\bar{M}}{L} \frac{\left(\delta^{-\frac{1}{2}} L\left(t_{k}-m N\right)\right)^{2}}{2 !} \\
\left\|\frac{2 u^{k}-2 u^{k-1}}{\tau}\right\|_{H} \leq M_{m-1}+\widetilde{M}_{m-1}+\bar{M}\left(t_{k}-m N\right)+\frac{\bar{M}}{L \delta^{-\frac{1}{2}}} \frac{\left(\delta^{-\frac{1}{2}} L\left(t_{k}-m N\right)\right)^{2}}{2 !}
\end{gathered}
$$

for any $1+m N \leq k \leq(m+1) N$. Let

$$
\left\|n u^{k}-(n-1) u^{k}\right\|_{H} \leq \frac{\bar{M}}{L} \frac{\left(L \delta^{-\frac{1}{2}}\left(t_{k}-m N\right)\right)^{n}}{n !},
$$




$$
\begin{gathered}
\left\|\frac{n u^{k}-n u^{k-1}}{\tau}-\frac{(n-1) u^{k}-(n-1) u^{k-1}}{\tau}\right\|_{H} \leq \frac{\bar{M}}{L \delta^{-\frac{1}{2}}} \frac{\left(L \delta^{-\frac{1}{2}}\left(t_{k}-m N\right)\right)^{n}}{n !}, \\
\left\|n u^{k}\right\|_{H} \leq \delta^{-1}\left[M_{m-1}+\widetilde{M}_{m-1}\right]+\delta^{-\frac{1}{2}} \bar{M}\left(t_{k}-m N\right) \\
+\bar{M} \frac{\left(\delta^{-\frac{1}{2}} L\left(t_{k}-m N\right)\right)^{2}}{2 !}+\cdots+\frac{\bar{M}}{L} \frac{\left(L \delta^{-\frac{1}{2}}\left(t_{k}-m N\right)\right)^{n}}{n !}, \\
\left\|\frac{n u^{k}-n u^{k-1}}{\tau}\right\|_{H} \leq \delta^{-\frac{1}{2}}\left[M_{m-1}+\widetilde{M}_{m-1}\right]+\bar{M}\left(t_{k}-m N\right) \\
\quad+\frac{\bar{M}}{L \delta^{-\frac{1}{2}}} \frac{\left(\delta^{-\frac{1}{2}} L\left(t_{k}-m N\right)\right)^{2}}{2 !}+\cdots+\frac{\bar{M}}{L \delta^{-\frac{1}{2}}} \frac{\left(L \delta^{-\frac{1}{2}}\left(t_{k}-m N\right)\right)^{n}}{n !}
\end{gathered}
$$

for any $1+m N \leq k \leq(m+1) N$. Applying formula (39), and estimates (7), (32), and (33), we get

$$
\begin{gathered}
\left\|(n+1) u^{1}-n u^{1}\right\|_{H}=0, \\
\left\|(n+1) u^{k}-n u^{k}\right\|_{H} \leq\left\|A^{-\frac{1}{2}}\right\|_{H \rightarrow H} \sum_{p=m N+1}^{k-1} \frac{1}{2}\left[\|R\|_{H \rightarrow H}^{k-p}+\|\widetilde{R}\|_{H \rightarrow H}^{k-p}\right] \\
\left.\times \| f\left(t_{p}, n u^{p}, \frac{u^{p-N}-u^{p-N-1}}{\tau}, u^{p-N}\right)\right)-f\left(t_{p},(n-1) u^{p}, \frac{u^{p-N}-u^{p-N-1}}{\tau}, u^{p-N}\right) \|_{H} \tau \\
\leq \delta^{-\frac{1}{2}} \sum_{p=m N+1}^{k-1} L n u^{p}-(n-1) u^{p} \|_{H} \tau \leq \delta^{-\frac{1}{2}} L \sum_{p=m N+1}^{k-1} \frac{\bar{M}}{L} \frac{\left(L \delta^{-\frac{1}{2}} t_{p-m N}\right)^{n}}{n !} \tau \\
\leq \frac{\bar{M}}{L} \frac{\left(L \delta^{-\frac{1}{2}}\left(t_{k}-m N\right)\right)^{n+1}}{(n+1) !}, \\
\left\|\frac{(n+1) u^{1}-n u^{0}}{\tau}-\frac{n u^{1}-n u^{0}}{\tau}\right\|_{H}=0, \\
\left.\times \| f\left(t_{p}, n u^{p}, \frac{u^{p-N}-u^{p-N-1}}{\tau}, u^{p-N}\right)\right)-f\left(t_{p},(n-1) u^{p}, \frac{u^{p-N}-u^{p-N-1}}{\tau}, u^{p-N}\right) \|_{H} \tau \\
\left.\leq \sum_{p m N+1}^{k-1} L\left\|n u^{p}-(n-1) u^{p}\right\|_{H} \tau \leq \sum_{p=m N+1}^{k-1} \frac{1}{L} \frac{\bar{M}}{L \delta^{-\frac{1}{2}}} \frac{\left(L \delta^{-\frac{1}{2}} t_{p-m N}\right)^{n}}{n !} \tau\left\|_{H \rightarrow H}^{k-p}+\right\| \widetilde{R} \|_{H \rightarrow H}^{k-p}\right] \\
\leq \frac{\bar{M}}{L \delta^{-\frac{1}{2}}} \frac{\left(L \delta^{-\frac{1}{2}}\left(t_{k}-m N\right)\right)^{n+1}}{(n+1) !}
\end{gathered}
$$

for any $2+m N \leq k \leq(m+1) N$. Using the triangle inequality, we get

$$
\begin{gathered}
\left\|(n+1) u^{k}\right\|_{H} \leq \delta^{-\frac{1}{2}}\left[M_{m-1}+\widetilde{M}_{m-1}\right]+\delta^{-\frac{1}{2}} \bar{M}\left(t_{k}-m N\right) \\
+\bar{M} \frac{\left(\delta^{-\frac{1}{2}} L\left(t_{k}-m N\right)\right)^{2}}{2 !}+\cdots+\frac{\bar{M}}{L} \frac{\left(L \delta^{-\frac{1}{2}}\left(t_{k}-m N\right)\right)^{n+1}}{(n+1) !}, \\
\left\|\frac{(n+1) u^{k}-(n+1) u^{k-1}}{\tau}\right\|_{H} \leq M_{m-1}+\widetilde{M}_{m-1}+\bar{M}\left(t_{k}-m N\right)
\end{gathered}
$$




$$
+\frac{\bar{M}}{L \delta^{-\frac{1}{2}}} \frac{\left(\delta^{-\frac{1}{2}} L\left(t_{k}-m N\right)\right)^{2}}{2 !}+\cdots+\frac{\bar{M}}{L \delta^{-\frac{1}{2}}} \frac{\left(L \delta^{-\frac{1}{2}}\left(t_{k}-m N\right)\right)^{n+1}}{(n+1) !}
$$

for any $1+m N \leq k \leq(m+1) N$. Therefore, for any $n, n \geq 1$, we have that

$$
\begin{gathered}
\left\|(n+1) u^{k}-n u^{k}\right\|_{H} \leq \frac{\bar{M}}{L} \frac{\left(L \delta^{-\frac{1}{2}}\left(t_{k}-m N\right)\right)^{n+1}}{(n+1) !} \\
\left\|\frac{(n+1) u^{k}-(n+1) u^{k-1}}{\tau}-\frac{n u^{k}-n u^{k-1}}{\tau}\right\|_{H} \leq \frac{\bar{M}}{L \delta^{-\frac{1}{2}}} \frac{\left(L \delta^{-\frac{1}{2}}\left(t_{k}-m N\right)\right)^{n+1}}{(n+1) !}
\end{gathered}
$$

and

$$
\begin{gathered}
\left\|(n+1) u^{k}\right\|_{H} \leq \delta^{-\frac{1}{2}}\left[M_{m-1}+\widetilde{M}_{m-1}\right]+\delta^{-\frac{1}{2}} \bar{M}\left(t_{k}-m N\right) \\
+\frac{\bar{M}}{L} \frac{\left(\delta^{-\frac{1}{2}} L\left(t_{k}-m N\right)\right)^{2}}{2 !}+\cdots+\frac{\bar{M}}{L} \frac{\left(L \delta^{-\frac{1}{2}}\left(t_{k}-m N\right)\right)^{n+1}}{(n+1) !} \\
\left\|\frac{(n+1) u^{k}-(n+1) u^{k-1}}{\tau}\right\|_{H} \leq M_{m-1}+\widetilde{M}_{m-1}+\bar{M}\left(t_{k}-m N\right) \\
+\frac{\bar{M}}{L \delta^{-\frac{1}{2}}} \frac{\left(\delta^{-\frac{1}{2}} L\left(t_{k}-m N\right)\right)^{2}}{2 !}+\cdots+\frac{\bar{M}}{L \delta^{-\frac{1}{2}}} \frac{\left(L \delta^{-\frac{1}{2}}\left(t_{k}-m N\right)\right)^{n+1}}{(n+1) !}
\end{gathered}
$$

for any $1+m N \leq k \leq(m+1) N$ by mathematical induction. From that and formula (37) it follows that

$$
\begin{aligned}
& \left\|u^{k}\right\|_{H} \leq\left\|0 u^{k}\right\|_{H}+\sum_{i=0}^{\infty}\left\|(i+1) u^{k}-i u^{k}\right\|_{H} \\
& \leq \delta^{-\frac{1}{2}}\left[M_{m-1}+\widetilde{M}_{m-1}\right]+\sum_{i=0}^{\infty} \frac{\bar{M}}{L} \frac{\left(\delta^{-\frac{1}{2}} L\left(t_{k}-m N\right)\right)^{i+1}}{(i+1) !} \\
& \leq \delta^{-\frac{1}{2}}\left[M_{m-1}+\widetilde{M}_{m-1}\right]+\frac{\bar{M}}{L} e^{\delta^{-\frac{1}{2}} L\left(t_{k}-m N\right)}, \\
& \left\|\frac{u^{k}-u^{k-1}}{\tau}\right\|_{H} \leq\left\|\frac{0 u^{k}-0 u^{k-1}}{\tau}\right\|_{H}+\sum_{i=0}^{\infty}\left\|\frac{(i+1) u^{k}-(i+1) u^{k-1}}{\tau}-\frac{i u^{k}-i u^{k-1}}{\tau}\right\|_{H} \\
& \leq M_{m-1}+\widetilde{M}_{m-1}+\sum_{i=0}^{\infty} \frac{\bar{M}}{L \delta^{-\frac{1}{2}}} \frac{\left(L \delta^{-\frac{1}{2}}\left(t_{k}-m N\right)\right)^{i+1}}{(i+1) !} \\
& \leq M_{m-1}+\widetilde{M}_{m-1}+\frac{\bar{M}}{L \delta^{-\frac{1}{2}}} e^{\delta^{-\frac{1}{2}} L\left(t_{k}-m N\right)}, \\
& \left\|A^{\frac{1}{2}} u^{k}\right\|_{H} \leq\left\|A^{\frac{1}{2}} 0 u^{k}\right\|_{H}+\sum_{i=0}^{\infty}\left\|A^{\frac{1}{2}}(i+1) u^{k}-A^{\frac{1}{2}} i u^{k}\right\|_{H} \leq M_{m-1}+\tilde{M}_{m-1} \\
& +\sum_{i=0}^{\infty} \frac{\bar{M}}{L \delta^{-\frac{1}{2}}} \frac{\left(L \delta^{-\frac{1}{2}}\left(t_{k}-m N\right)\right)^{i+1}}{(i+1) !} \leq M_{m-1}+\widetilde{M}_{m-1}+\frac{\bar{M}}{L \delta^{-\frac{1}{2}}} e^{\delta^{-\frac{1}{2}} L\left(t_{k}-m N\right)}
\end{aligned}
$$

for any $1+m N \leq k \leq(m+1) N$ which proves the existence of a bounded solution of difference scheme (28) in $[m \omega,(m+1) w]_{\tau} \times H$ of uniformly with respect to $\tau$. 
Step 3. Uniqueness of solution of difference scheme (28). We prove uniqueness of the uniformly bounded solution of problem (28). Suppose that there is a bounded solution $v^{\tau}=\left\{v^{k}\right\}_{k=0}^{\infty}$ of problem (28) and $v^{\tau} \neq u^{\tau}$. Denoting $z^{\tau}=v^{\tau}-u^{\tau}$ and using Equation (28), we get

$$
\left\{\begin{array}{l}
\frac{z^{k+1}-2 z^{k}+z^{k-1}}{\tau^{2}}+A z^{k+1} \\
=f\left(t_{k}, v^{k}, \frac{v^{k-N}-v^{k-N-1}}{\tau}, v^{k-N}\right)-f\left(t_{k}, u^{k}, \frac{u^{k-N}-u^{k-N-1}}{\tau}, u^{k-N}\right), k \geq 1, \\
\left(I+\tau^{2} A\right) \frac{z^{k+1}-z^{k}}{\tau}=\frac{z^{k}-z^{k-1}}{\tau}, k=m N, m=1, \ldots, \\
z^{k}=0,-N \leq k \leq 0
\end{array}\right.
$$

for $z^{\tau}$. We consider the interval $1 \leq k \leq N$. Since $v^{k-N}=u^{k-N}=\varphi\left(t_{k-N}\right)$, we have that

$$
\left\{\begin{array}{l}
\frac{z^{k+1}-2 z^{k}+z^{k-1}}{\tau^{2}}+A z^{k+1} \\
=f\left(t_{k}, v^{k}, \frac{\varphi_{k-N}-\varphi_{k-N-1}}{\tau}, \varphi_{k-N}\right)-f\left(t_{k}, u^{k}, \frac{\varphi_{k-N}-\varphi_{k-N-1}}{\tau}, \varphi_{k-N}\right), \quad 1 \leq k \leq N, \\
\left(I+\tau^{2} A\right) z^{1}=0, z^{0}=0 .
\end{array}\right.
$$

Therefore,

$$
\begin{gathered}
\left\|z^{k}\right\|_{H} \leq\left\|A^{-\frac{1}{2}}\right\|_{H \rightarrow H} \sum_{p=1}^{k-1} \frac{1}{2}\left[\|R\|_{H \rightarrow H}^{k-p}+\|\widetilde{R}\|_{H \rightarrow H}^{k-p}\right] \\
\times\left\|f\left(t_{k}, v^{k}, \frac{\varphi_{k-N}-\varphi_{k-N-1}}{\tau}, \varphi_{k-N}\right)-f\left(t_{k}, u^{k}, \frac{\varphi_{k-N}-\varphi_{k-N-1}}{\tau}, \varphi_{k-N}\right)\right\|_{H} \tau .
\end{gathered}
$$

Applying estimates estimates (7), (32), and (33), we get

$$
\left\|z^{k}\right\|_{H} \leq \delta^{-\frac{1}{2}} L \sum_{m=1}^{k-1}\left\|z^{m}\right\|_{H} \tau
$$

Using the discrete analogy of the integral inequality, we get

$$
\left\|z^{k}\right\|_{H} \leq 0
$$

From that it follows that $z^{k}=0,1 \leq k \leq N$ which proves the uniqueness of a bounded solution of problem (28) in $[0, w]_{\tau} \times H$ of uniformly with respect to $\tau$. Using the same method and mathematical induction, we can prove the uniqueness of a bounded solution of problem (28) in $[m \omega,(m+1) w]_{\tau} \times H$ of uniformly with respect to $\tau$. Theorem 8 is proved.

\section{Applications of Theorem 8}

First, we consider the initial value problem (17) for the one dimensional semininear hyperbolic differential equation with time delay term and with nonlocal conditions.

The discretization of problem (17) is provided in two steps. To the differential operator $A$ generated by problem (17), we assign the difference operator $A_{h}^{x}$ by the formula

$$
A_{h}^{x} \varphi_{h}(x)=\left\{-\left(a(x) \varphi_{\bar{x}}\right)_{x, r}+\delta \varphi_{r}(x)\right\}_{1}^{K-1},
$$


acting in the space of grid functions $\varphi_{h}(x)=\left\{\varphi_{r}\right\}_{0}^{K}$ satisfying the conditions $\varphi_{0}=\varphi_{K}, \varphi_{1}-\varphi_{0}=$ $\varphi_{K}-\varphi_{K-1}$. It is known that $A_{h}^{x}$ is a self-adjoint positive definite operator in $L_{2 h}=L_{2}\left([0, l]_{h}\right)[51]$. With the help of $A_{h}^{x}$, we arrive at the initial value problem

$$
\left\{\begin{array}{l}
\frac{d^{2} u^{h}(t, x)}{d t^{2}}+A_{h}^{x} u^{h}(t, x)=f^{h}\left(t, x, u^{h}(t, x), u_{t}^{h}(t-w, x), u^{h}(t-w, x)\right), \\
0<t<\infty, x \in[0, l]_{h} \\
u^{h}(t, x)=\varphi^{h}(t, x), \quad-\omega \leq t \leq 0, x \in[0, l]_{h} .
\end{array}\right.
$$

In the second step, we replace problem (42) by first order of accuracy difference scheme (28)

$$
\left\{\begin{array}{l}
\frac{u_{k+1}^{h}(x)-2 u_{k}^{h}(x)+u_{k-1}^{h}(x)}{\tau^{2}}+A_{h}^{x} u_{k+1}^{h}(x)=f\left(t_{k}, x, u_{k}^{h}(x), \frac{u_{k-N}^{h}(x)-u_{k-N-1}^{h}(x)}{\tau}, u_{k-N}^{h}(x)\right), \\
t_{k}=k \tau, 1 \leq k<\infty, N \tau=\omega \\
\left(I+\tau^{2} A_{h}^{x}\right) \frac{u_{k+1}^{h}(x)-u_{k}^{h}(x)}{\tau}=\frac{u_{k}^{h}(x)-u_{k-1}^{h}(x)}{\tau}, k=m N, m=0,1, \ldots \\
u_{k}^{h}(x)=\varphi^{h}\left(t_{k}, x\right), t_{k}=k \tau,-N \leq k \leq 0, x \in[0, l]_{h} .
\end{array}\right.
$$

Theorem 9. Suppose that assumptions of Theorem 2 hold. Then, there exists a unique solution $\left\{u_{k}^{h}\right\}_{k=0}^{\infty}$ of difference scheme (43) which is bounded in $[m \omega,(m+1) w]_{\tau} \times L_{2 h}, m=0,1, \cdots$ of uniformly with respect to $\tau$ and $h$.

Proof. Difference scheme (43) can be written in abstract form (28) in a Hilbert space $L_{2 h}=L_{2}\left([0, l]_{h}\right)$ with self-adjoint positive definite operator $A_{h}=A_{h}^{x}$ by formula (41). Here,

$f\left(t_{k}, x, u_{k}^{h}(x), \frac{u_{k-N}^{h}(x)-u_{k-N-1}^{h}(x)}{\tau}, u_{k-N}^{h}(x)\right)$ and $u_{k}^{h}=u_{k}^{h}(x)$ are abstract mesh functions defined on $[0, l]_{h}$ with the values in $H=L_{2 h}$. Therefore, the proof of Theorem 9 is based on Theorem 8 and symmetry properties of the difference operator $A_{h}^{x}$.

Second, we study the initial nonlocal boundary value problem (22) for one dimensional semilinear delay hyperbolic equations type with involution. The discretization of problem (22) is provided in two steps. To the differential operator $A$ generated by problem (22), we assign the difference operator $A_{h}^{x}$ by the formula

$$
A_{h}^{x} \varphi^{h}(x)=\left\{-\left(a(x) \varphi_{\bar{x}}(x)\right)_{x, r}-\beta\left(a(-x) \varphi_{\bar{x}}(-x)\right)_{x, r}+\delta \varphi^{r}(x)\right\}_{-M+1}^{M-1},
$$

acting in the space of grid functions $\varphi^{h}(x)=\left\{\varphi^{r}\right\}_{-M}^{M}$ satisfying the conditions $\varphi^{-M}=\varphi^{M}=0$. It is known that $A_{h}^{x}$ is a self-adjoint positive definite operator in $L_{2 h}=L_{2}\left([-l, l]_{h}\right)$ [52]. With the help of $A_{h}^{x}$, we arrive at the initial value problem

$$
\left\{\begin{array}{l}
\frac{d^{2} u^{h}(t, x)}{d t^{2}}+A_{h}^{x} u^{h}(t, x)=f^{h}\left(t, x, u^{h}(t, x), u_{t}^{h}(t-w, x), u^{h}(t-w, x)\right), \\
0<t<\infty, x \in[-l, l]_{h} \\
u^{h}(t, x)=\varphi^{h}(t, x), \quad-\omega \leq t \leq 0, x \in[-l, l]_{h} .
\end{array}\right.
$$


In the second step, we replace problem (45) by first order of accuracy difference scheme (28)

$$
\left\{\begin{array}{l}
\frac{u_{k+1}^{h}(x)-2 u_{k}^{h}(x)+u_{k-1}^{h}(x)}{\tau^{2}}+A_{h}^{x} u_{k+1}^{h}(x)=f\left(t_{k}, x, u_{k}^{h}(x), \frac{u_{k-N}^{h}(x)-u_{k-N-1}^{h}(x)}{\tau}, u_{k-N}^{h}(x)\right), \\
t_{k}=k \tau, 1 \leq k<\infty, N \tau=\omega, \\
\left(I+\tau^{2} A_{h}^{x}\right) \frac{u_{k+1}^{h}(x)-u_{k}^{h}(x)}{\tau}=\frac{u_{k}^{h}(x)-u_{k-1}^{h}(x)}{\tau}, k=m N, \quad m=0,1, \ldots, \\
u_{k}^{h}(x)=\varphi^{h}\left(t_{k}, x\right), \quad t_{k}=k \tau,-N \leq k \leq 0, x \in[-l, l]_{h} .
\end{array}\right.
$$

Theorem 10. Suppose that assumptions of Theorem 3 hold. Then, there exists a unique solution $\left\{u_{k}^{h}\right\}_{k=0}^{\infty}$ of difference scheme (46) which is bounded in $[m \omega,(m+1) w]_{\tau} \times L_{2 h}, m=0,1, \cdots$ uniformly with respect to $\tau$ and $h$.

Proof. Difference scheme (46) can be written in abstract form (28) in a Hilbert space $L_{2 h}=L_{2}\left([-l, l]_{h}\right)$ with self-adjoint positive definite operator $A_{h}=A_{h}^{x}$ by formula (44). Here,

$f\left(t_{k}, x, u_{k}^{h}(x), \frac{u_{k-N}^{h}(x)-u_{k-N-1}^{h}(x)}{\tau}, u_{k-N}^{h}(x)\right)$ and $u_{k}^{h}=u_{k}^{h}(x)$ are abstract mesh functions defined on $[-l, l]_{h}$ with the values in $H=L_{2 h}$. Therefore, the proof of Theorem 10 is based on Theorem 8 and symmetry properties of the difference operator $A_{h}^{x}$.

Third, we study the initial boundary value problem (23) for multidimensional semilinear delay hyperbolic equations.

The discretization of problem (23) is provided in two steps. In the first step, here and in future, we define the grid space

$$
\begin{gathered}
\bar{\Omega}_{h}=\left\{x=x_{r}=\left(h_{1} j_{1}, \cdots, h_{m} j_{m}\right), j=\left(j_{1}, \cdots, j_{m}\right), 0 \leq j_{r} \leq N_{r},\right. \\
\left.N_{r} h_{r}=1, \quad r=1, \cdots, m\right\}, \Omega_{h}=\bar{\Omega}_{h} \cap \Omega, S_{h}=\bar{\Omega}_{h} \cap S .
\end{gathered}
$$

We introduce the Banach space $L_{2 h}=L_{2}\left(\bar{\Omega}_{h}\right)$ of the grid functions $\varphi_{h}(x)=\left\{\varphi\left(h_{1} r_{1}, \ldots, h_{m} r_{m}\right)\right\}$ defined on $\bar{\Omega}_{h}$, equipped with the norm

$$
\left\|\varphi_{h}\right\|_{L_{2 h}}=\left(\sum_{x \in \bar{\Omega}_{h}}\left|\varphi_{h}(x)\right|^{2} h_{1} \cdots h_{m}\right)^{1 / 2}
$$

to the differential operator $A$ generated by problem (23), we assign the difference operator $A_{h}^{x}$ by the formula

$$
A_{h}^{x} u_{h}(x)=-\sum_{r=1}^{m}\left(a_{r}(x) u_{\bar{x}_{r}, h}\right)_{x_{r}, j_{r}}
$$

acting in the space of grid functions $u_{h}(x)$, satisfying the conditions $u_{h}(x)=0\left(\forall x \in S_{h}\right)$. It is known that $A_{h}^{x}$ is a self-adjoint positive definite operator in $L_{2 h}$. With the help of $A_{h^{\prime}}^{x}$ we arrive at the initial value problem

$$
\left\{\begin{array}{l}
\frac{d^{2} u_{h}(t, x)}{d t^{2}}+A_{h}^{x} u_{h}(t, x)=f^{h}\left(t, x, u^{h}(t, x), u_{t}^{h}(t-w, x), u^{h}(t-w, x)\right), \\
0<t<\infty, \quad x \in \Omega_{h}, \\
u_{h}(t, x)=\varphi_{h}(t, x), \quad-\omega \leq t \leq 0, \quad x \in \bar{\Omega}_{h} .
\end{array}\right.
$$


In the second step, we replace problem (48) by first order of accuracy difference scheme (28)

$$
\left\{\begin{array}{l}
\frac{u_{h}^{k+1}(x)-2 u_{h}^{k}(x)+u_{h}^{k-1}(x)}{\tau^{2}}+A_{h}^{x} u_{h}^{k+1}(x)=f\left(t_{k}, x, u_{k}^{h}(x), \frac{u_{k-N}^{h}(x)-u_{k-N-1}^{h}(x)}{\tau}, u_{k-N}^{h}(x)\right), \\
t_{k}=k \tau, 1 \leq k<\infty, N \tau=\omega, x \in \Omega_{h}, \\
\left(I+\tau^{2} A_{h}^{x}\right) \frac{u_{h}^{k+1}(x)-u_{h}^{k}(x)}{\tau}=\frac{u_{h}^{k}(x)-u_{h}^{k-1}(x)}{\tau}, k=m N, m=0,1, \ldots, \\
u_{h}^{k}(x)=\varphi_{h}\left(t_{k}, x\right), t_{k}=k \tau,-N \leq k \leq 0, x \in \bar{\Omega}_{h} .
\end{array}\right.
$$

Theorem 11. Suppose that assumptions of Theorem 4 hold. Then, there exists a unique solution $\left\{u_{k}^{h}\right\}_{k=0}^{\infty}$ of difference scheme (49) which is bounded in $[m \omega,(m+1) w]_{\tau} \times L_{2 h}, m=0,1, \cdots$ uniformly with respect to $\tau$ and $h$.

Proof. Difference scheme (49) can be written in abstract form (28) in a Hilbert space $L_{2 h}=$ $L_{2}\left(\bar{\Omega}_{h}\right)$ with self-adjoint positive definite operator $A_{h}=A_{h}^{x}$ by formula (47). Here, $f\left(t_{k}, x, u_{k}^{h}(x), \frac{u_{k-N}^{h}(x)-u_{k-N-1}^{h}(x)}{\tau}, u_{k-N}^{h}(x)\right)$ and $u_{k}^{h}=u_{k}^{h}(x)$ are abstract mesh functions defined on $\bar{\Omega}_{h}$ with the values in $H=L_{2 h}$. Therefore, the proof of Theorems 11 is based on the abstract Theorem 8 and symmetry properties of the difference operator $A_{h}^{x}$ defined by formula (47) and the following theorem on coercivity inequality for the solution of the elliptic problem in $L_{2 h}$ [53].

Theorem 12. For the solutions of the elliptic difference problem

$$
\left\{\begin{array}{l}
A_{h}^{x} u^{h}(x)=\omega^{h}(x), x \in \Omega_{h}, \\
u^{h}(x)=0, x \in S_{h},
\end{array}\right.
$$

the coercivity inequality

$$
\sum_{r=1}^{n}\left\|u^{h} x_{r} x_{\bar{r}}\right\|_{L_{2 h}} \leq M_{1}\left\|\omega^{h}\right\|_{L_{2 h}}
$$

is satisfied, where $M_{1}$ does not depend on $h$ and $\omega^{h}$.

Fourth, we study the initial boundary value problem (26) for multidimensional semilinear delay hyperbolic equations. The discretization of problem (23) is provided in two steps. To the differential operator $A$ generated by problem (26), we assign the difference operator $A_{h}^{x}$ by the formula

$$
A_{h}^{x} u^{h}(x)=-\sum_{r=1}^{m}\left(a_{r}(x) u_{\bar{x}_{r}}^{h}\right)_{x_{r}, j_{r}}+\delta u^{h}(x)
$$

acting in the space of grid functions $u^{h}(x)$, satisfying the conditions $D^{h} u^{h}(x)=0\left(\forall x \in S_{h}\right)$. Here $D^{h}$ is the approximation of operator $\frac{\partial}{\partial \vec{p}}$. With the help of $A_{h}^{x}$, we arrive at the initial value problem (48). In the second step, we replace problem (48) by first order of accuracy difference scheme (28), we get Equation (49). 
Theorem 13. Suppose that assumptions of Theorem 6 hold. Then, there exists a unique solution $\left\{u_{k}^{h}\right\}_{k=0}^{\infty}$ of difference scheme (49) which is bounded in $[m \omega,(m+1) w]_{\tau} \times L_{2 h}, m=0,1, \cdots$ uniformly with respect to $\tau$ and $h$.

Proof. Difference scheme (49) can be written in abstract form (28) in a Hilbert space $L_{2 h}$ $=L_{2}\left(\bar{\Omega}_{h}\right)$ with self-adjoint positive definite operator $A_{h}=A_{h}^{x}$ by formula (50). Here, $f\left(t_{k}, x, u_{k}^{h}(x), \frac{u_{k-N}^{h}(x)-u_{k-N-1}^{h}(x)}{\tau}, u_{k-N}^{h}(x)\right)$ and $u_{k}^{h}=u_{k}^{h}(x)$ are abstract mesh functions defined on $\bar{\Omega}_{h}$ with the values in $H=L_{2 h}$. Therefore, the proof of Theorems 13 is based on the abstract Theorem 8 and symmetry properties of the difference operator $A_{h}^{x}$ defined by formula (50) and the following theorem on coercivity inequality for the solution of the elliptic problem in $L_{2 h}$ [53].

Theorem 14. For the solutions of the elliptic difference problem

$$
\left\{\begin{array}{l}
A_{h}^{x} u^{h}(x)=\omega^{h}(x), x \in \Omega_{h}, \\
D^{h} u^{h}(x)=0, x \in S_{h}
\end{array}\right.
$$

the coercivity inequality

$$
\sum_{r=1}^{n}\left\|u^{h}{ }_{x_{r} x_{\bar{r}}}\right\|_{L_{2 h}} \leq M_{2}\left\|\omega^{h}\right\|_{L_{2 h}}
$$

is satisfied, where $M_{2}$ does not depend on $h$ and $\omega^{h}$.

\section{Numerical Experiments}

In general, it is not able to get the exact solution of semilinear hyperbolic problems. Therefore, numerical results for the solution of difference schemes for one and two dimensional semilinear hyperbolic equations with time delay are presented. These results fit with the theoretical results perfectly.

\subsection{One Dimensional Case}

For the numerical experiment, we consider the mixed problem

$$
\left\{\begin{array}{l}
\frac{\partial^{2} u(t, x)}{\partial t^{2}}-\frac{\partial^{2} u(t, x)}{\partial x^{2}}=2 e^{-t} \sin x+\cos (u(t, x) u(t-1, x)) \\
-\cos \left(e^{-t} \sin x u(t-1, x)\right), 0<t<\infty, 0<x<2 \pi \\
u(t, x)=e^{-t} \sin x, 0 \leq x \leq 2 \pi,-1 \leq t \leq 0 \\
u(t, 0)=u(t, 2 \pi), u_{x}(t, 0)=u_{x}(t, 2 \pi), t \geq 0
\end{array}\right.
$$


for the semilinear delay one dimensional hyperbolic differential equation with nonlocal boundary conditions. The exact solution of problem (51) is $u(t, x)=e^{-t} \sin x$. We will consider the following iterative difference scheme of first order of approximation in $t$ for the numerical solution of problem (51)

$$
\left\{\begin{array}{l}
\frac{{ }_{j} u_{n}^{k+1}-2\left({ }_{j} u_{n}^{k}\right)+{ }_{j} u_{n}^{k-1}}{\tau^{2}}-\frac{{ }_{j} u_{n+1}^{k+1}-2\left({ }_{j} u_{n}^{k+1}\right)+{ }_{j} u_{n-1}^{k+1}}{h^{2}}=2 e^{-t_{k}} \sin x_{n} \\
+\cos \left(\left({ }_{j}-1 u_{n}^{k}\right) u_{n}^{k-N}\right)-\cos \left(e^{-t_{k}} \sin x_{n}\left(u_{n}^{k-N}\right)\right), \\
t_{k}=k \tau, \quad x_{n}=n h, 1 \leq k<\infty, 1 \leq n \leq M-1, N \tau=1, \quad M h=2 \pi, \\
\frac{{ }_{j} u_{n}^{k+1}-{ }_{j} u_{n}^{k}}{\tau}-\frac{\tau}{h^{2}}\left({ }_{j} u_{n+1}^{k+1}-{ }_{j} u_{n+1}^{k}-2\left({ }_{j} u_{n}^{k+1}-{ }_{j} u_{n}^{k}\right)+{ }_{j} u_{n-1}^{k+1}-{ }_{j} u_{n-1}^{k}\right) \\
=\frac{u_{n}^{k}-u_{n}^{k-1}}{\tau}, k=m N+1, m=0,1, \ldots, k \geq 1, \\
u_{n}^{k}=e^{-t_{k}} \sin x_{n}, \quad t_{k}=k \tau, \quad x_{n}=n h, 0 \leq n \leq M, \quad-N \leq k \leq 0, \\
{ }_{j} u_{0}^{k}={ }_{j} u_{M}^{k}{ }_{j} u_{1}^{k}-{ }_{j} u_{0}^{k}={ }_{j} u_{M}^{k}-{ }_{j} u_{M-1}^{k}, 0 \leq k<\infty, \quad j=1,2, \ldots
\end{array}\right.
$$

for the semilinear delay hyperbolic equation. Here and in future $j$ denotes the iteration index and an initial guess $u_{n}^{k}, k \geq 1,0 \leq n \leq M$ is to be made.

For solving difference scheme (52), the numerical steps are given below. For $0 \leq k<N$, $0 \leq n \leq M$ the algorithm is as follows :

1. $j=1$.

2. $j-1 u_{n}^{k}$ is known.

3. $j u_{n}^{k}$ is calculated.

4. If the max absolute error between ${ }_{j-1} u_{n}^{k}$ and ${ }_{j} u_{n}^{k}$ is greater than the given tolerance value, take $j=j+1$ and go to step 2. Otherwise, terminate the iteration process and take $j u_{n}^{k}$ as the result of the given problem.

We write (52) in matrix form

$$
\begin{gathered}
A\left({ }_{j} u^{k+1}\right)+B\left({ }_{j} u^{k}\right)+C\left({ }_{j} u^{k-1}\right)=R \varphi\left({ }_{j-1} u^{k}, u^{k-N}\right), 1 \leq k<\infty, \\
u^{k}=\left\{e^{-t_{k}} \sin x_{n}\right\}_{n=0}^{M},-N \leq k \leq 0, \\
\frac{j^{j} u^{k+1}-j_{j} u^{k}}{\tau}-\left\{\frac{\tau}{h^{2}}\left({ }_{j} u_{n+1}^{k+1}-{ }_{j} u_{n+1}^{k}-2\left({ }_{j} u_{n}^{k+1}-{ }_{j} u_{n}^{k}\right)+{ }_{j} u_{n-1}^{k+1}-{ }_{j} u_{n-1}^{k}\right)\right\}_{n=1}^{M-1} \\
=\frac{u^{k}-u^{k-1}}{\tau}, k=m N+1, k \geq 1 .
\end{gathered}
$$

Here $R, A, B$, and $C$ are $(M+1) \times(M+1)$ matrices given below:

$$
R=\left[\begin{array}{cccccccc}
1 & 0 & 0 & 0 & 0 & . & 0 & 0 \\
0 & 1 & 0 & 0 & 0 & . & 0 & 0 \\
0 & 0 & 1 & 0 & 0 & . & 0 & 0 \\
. & . & . & . & . & . & . & . \\
0 & 0 & 0 & 0 & 0 & . & 1 & 0 \\
0 & 0 & 0 & 0 & 0 & . & 0 & 1
\end{array}\right]
$$




$$
\begin{aligned}
A & =\left[\begin{array}{cccccccc}
1 & 0 & 0 & 0 & 0 & . & 0 & -1 \\
a & b & a & 0 & 0 & . & 0 & 0 \\
0 & a & b & a & 0 & . & 0 & 0 \\
0 & 0 & a & b & a & . & 0 & 0 \\
0 & 0 & 0 & a & b & . & 0 & 0 \\
. & . & . & . & . & . & . & . \\
0 & 0 & 0 & 0 & 0 & . & b & a \\
1 & -1 & 0 & 0 & 0 & . & -1 & 1
\end{array}\right], \\
B & =\left[\begin{array}{cccccccc}
0 & 0 & 0 & 0 & 0 & . & 0 & 0 \\
0 & c & 0 & 0 & 0 & . & 0 & 0 \\
0 & 0 & c & 0 & 0 & . & 0 & 0 \\
0 & 0 & 0 & c & 0 & . & 0 & 0 \\
. & . & . & . & . & . & . & . \\
0 & 0 & 0 & 0 & 0 & . & c & 0 \\
0 & 0 & 0 & 0 & 0 & . & 0 & 0
\end{array}\right], \\
C & =\left[\begin{array}{cccccccc}
0 & 0 & 0 & 0 & 0 & . & 0 & 0 \\
0 & d & 0 & 0 & 0 & . & 0 & 0 \\
0 & 0 & d & 0 & 0 & . & 0 & 0 \\
0 & 0 & 0 & d & 0 & . & 0 & 0 \\
. & . & . & . & . & . & . & . \\
0 & 0 & 0 & 0 & 0 & . & d & 0 \\
0 & 0 & 0 & 0 & 0 & . & 0 & 0
\end{array}\right]
\end{aligned}
$$

where

$$
a=-\frac{1}{h^{2}}, b=\frac{1}{\tau^{2}}+\frac{2}{h^{2}}, c=-\frac{2}{\tau^{2}}, d=\frac{1}{\tau^{2}} .
$$

Finally, here $\varphi\left({ }_{j-1} u^{k}, u^{k-N}\right)$ and ${ }_{j} u^{s}, s=k, k \pm 1$ are $(M+1) \times 1$ column vectors as

$$
\begin{gathered}
\varphi\left({ }_{j-1} u^{k}, u^{k-N}\right)=\left[\begin{array}{c}
0 \\
j \varphi_{1}^{k} \\
\cdot \\
j \varphi_{M-1}^{k} \\
0
\end{array}\right],{ }_{j} u^{s}=\left[\begin{array}{c}
{ }_{j} u_{0}^{s} \\
j \\
j \\
\cdot \\
j u_{M-1}^{s} \\
j u_{M}^{s}
\end{array}\right], \\
j \varphi_{n}^{k}=2 e^{-t_{k}} \sin x_{n}+\cos \left(\left({ }_{j-1} u_{n}^{k}\right)\left(u_{n}^{k-N}\right)\right)-\cos \left(e^{-t_{k}} \sin x_{n} u_{n}^{k-N}\right)
\end{gathered}
$$

for $1 \leq k \leq N-1,1 \leq n \leq M-1$.

So, we have the initial value problem for the second order difference equation with respect to $k$ with matrix coefficients. From Equations (53) and (54) it follows that

$$
\begin{gathered}
{ }_{j} u^{k+1}=-A^{-1}\left(B_{j} u^{k}-C_{j} u^{k-1}+A^{-1} R \varphi^{k}\left({ }_{j-1} u^{k}, u^{k-N}\right)\right), \\
{ }_{j} u^{k}=\left\{e^{-t_{k}} \sin x_{n}\right\}_{n=0}^{M}, \quad-N \leq k \leq 0, \\
{ }_{j} u^{k+1}=\psi\left(u^{k}, u^{k-1}\right), \quad k=m N+1, m=0,1, \ldots, k \geq 1 .
\end{gathered}
$$

Here, $\psi\left(u^{k}, u^{k-1}\right)$ is $(M+1) \times 1$ column vector defined by formula (54). 
In computations the initial guess is chosen as $0 u^{k}=\left\{\sin x_{n}\right\}_{n=0}^{M}$ and when the maximum errors between two consecutive results of iterative difference scheme (52) become less than $10^{-8}$, the iterative process is terminated. We present numerical experimental results for different values of $N$ and $M$ and $u_{n}^{k}$ represent the numerical solutions of difference scheme (52) at $\left(t_{k}, x_{n}\right)$. The table of numerical results is constructed for $N=M=30,60,120$ in $t \in[0,1], t \in[1,2], t \in[2,3]$, respectively and the errors are computed by the following formula

$$
m E_{M}^{N}=\max _{m N+1 \leq k \leq(m+1) N, 0 \leq n \leq M}\left|u\left(t_{k}, x_{n}\right)-{ }_{j} u_{n}^{k}\right| .
$$

As can be seen from tables, these numerical experiments support the theoretical statements. The number of iterations and maximum errors are decreasing with the increase of grid points.

In Table 1 , as we increase values of $M$ and $N$ each time starting from $M=N=30$ by a factor of 2 the errors in the first order of accuracy difference scheme decrease approximately by a factor of $1 / 2$. The errors presented in Table 1 indicate the first order of accuracy of the difference scheme.

Table 1. The errors (52) (Number of the iteration $=j$ ).

\begin{tabular}{cccc}
\hline$m E_{M}^{N} /(\boldsymbol{N}, \boldsymbol{M})$ & $\mathbf{( 3 0 , 3 0 )}$ & $\mathbf{( 6 0 , 6 0 )}$ & $\mathbf{( 1 2 0 , 1 2 0 )}$ \\
\hline in $t \in[0,1]$ & $4.1195 \times 10^{-3}, j=6$ & $2.0322 \times 10^{-3}, j=6$ & $1.0098 \times 10^{-3}, j=6$ \\
\hline in $t \in[1,2]$ & $2.3014 \times 10^{-3}, j=6$ & $1.1297 \times 10^{-3}, j=6$ & $5.6051 \times 10^{-4}, j=2$ \\
\hline in $t \in[2,3]$ & $1.0245 \times 10^{-3}, j=6$ & $5.0161 \times 10^{-4}, j=6$ & $2.4864 \times 10^{-4}, j=6$ \\
\hline
\end{tabular}

\subsection{Two-Dimensional Case}

For the numerical experiment, we consider the mixed boundary value problem

$$
\left\{\begin{array}{l}
\frac{\partial^{2} u(t, x, y)}{\partial t^{2}}-\frac{\partial^{2} u(t, x, y)}{\partial x^{2}}-\frac{\partial^{2} u(t, x, y)}{\partial y^{2}}=2 e^{-t} \sin x \sin y+\cos (u(t, x, y) u(t-1, x, y)) \\
-\cos \left(e^{-t} \sin x \sin y u(t-1, x, y)\right), 0<t<\infty, 0<x, y<\pi, \\
u(t, x, y)=e^{-t} \sin x \sin y, 0 \leq x, y \leq \pi,-1 \leq t \leq 0, \\
u(t, 0, y)=u(t, \pi, y)=0,0 \leq y \leq \pi, t \geq 0, \\
u(t, x, 0)=u(t, x, \pi)=0,0 \leq x \leq \pi, t \geq 0
\end{array}\right.
$$


for the semilinear two dimensional delay hyperbolic equation. The exact solution of problem (56) is $u(t, x)=e^{-t} \sin x \sin y$. We will consider the following iterative difference scheme of first order of approximation in $t$ for the numerical solution of the initial-boundary value problem (56)

$$
\left\{\begin{array}{l}
\frac{u_{n, i}^{k+1}-2\left({ }_{j} u_{n, i}^{k}\right)+{ }_{j} u_{n, i}^{k-1}}{\tau^{2}}-\frac{j u_{n+1, i}^{k+1}-2\left({ }_{j} u_{n, i}^{k+1}\right)+{ }_{j} u_{n-1, i}^{k+1}}{h^{2}}-\frac{{ }_{j} u_{n, i+1}^{k+1}-2\left({ }_{j} u_{n, i}^{k+1}\right)+{ }_{j} u_{n, i-1}^{k+1}}{h^{2}} \\
=2 e^{-t_{k}} \sin x_{n} \sin x_{i}+\cos \left(\left({ }_{j-1} u_{n, i}^{k}\right) u_{n, i}^{k-N}\right)-\cos \left(e^{-t_{k}} \sin x_{n} \sin x_{i} u_{n, i}^{k-N}\right), \\
t_{k}=k \tau, \quad x_{n}=n h, \quad 1 \leq k<\infty, \quad 1 \leq n, i \leq M-1, N \tau=1, \quad M h=\pi, \\
u_{n, i}^{k}=e^{-t_{k}} \sin x_{n} \sin x_{i}, t_{k}=k \tau, \quad x_{n}=n h, 0, i \leq M,-N \leq k \leq 0, \\
\frac{u_{n, i}^{k+1}-u_{n, i}^{k}}{\tau}-\frac{\tau}{h^{2}}\left({ }_{j} u_{n+1, i}^{k+1}-{ }_{j} u_{n+1, i}^{k}-2\left({ }_{j} u_{n, i}^{k+1}-{ }_{j} u_{n, i}^{k}\right)+{ }_{j} u_{n, i-1}^{k+1}-{ }_{j} u_{n, i-1}^{k}\right) \\
-\frac{\tau}{h^{2}}\left({ }_{j} u_{n, i+1}^{k+1}-{ }_{j} u_{n, i+1}^{k}-2\left({ }_{j} u_{n, i}^{k+1}-{ }_{j} u_{n, i}^{k}\right)+{ }_{j} u_{n-1, i}^{1}-{ }_{j} u_{n-1, i}^{k}\right) \\
=\frac{u_{n, i}^{k}-u_{n, i}^{k-1}}{\tau}, k=m N+1, m=0,1, \ldots, k \geq 1, \\
{ }_{j} u_{0, i}^{k}={ }_{j} u_{M, i}^{k}=0,0 \leq i \leq M{ }_{, j} u_{n, 0}^{k}={ }_{j} u_{n, M}^{k}=0,0 \leq n \leq M, \\
0 \leq k<\infty, j=1,2, \ldots
\end{array}\right.
$$

for the semilinear delay hyperbolic equation. Here and in the future $j$ denotes the iteration index and an initial guess $u_{n, i}^{k}, \quad k \geq 1,0 \leq n, i \leq M$ is to be made. For solving difference scheme (57), the numerical steps are given below. For $0 \leq k<N, 0 \leq n, i \leq M$ the algorithm is as follows :

1. $j=1$.

2. $j-1 u_{n, i}^{k}$ is known.

3. ${ }_{j} u_{n, i}^{k}$ is calculated.

4. If the max absolute error between ${ }_{j-1} u_{n, i}^{k}$ and ${ }_{j} u_{n, i}^{k}$ is greater than the given tolerance value, take $j=j+1$ and go to step 2. Otherwise, terminate the iteration process and take ${ }_{j} u_{n, i}^{k}$ as the result of the given problem.

We write Equation (57) in matrix form

$$
\begin{gathered}
A\left({ }_{j} u^{k+1}\right)+B\left({ }_{j} u^{k}\right)+C\left({ }_{j} u^{k-1}\right)=R \varphi\left({ }_{j}-1 u^{k}, u^{k-N}\right), 1 \leq k<\infty, \\
u^{k}=\left\{e^{-t_{k}} \sin x_{n} \sin x_{i}\right\}_{n, i=0}^{M},-N \leq k \leq 0, \\
\frac{{ }_{j} u^{k+1}-{ }_{j} u^{k}}{\tau}-\left\{\frac{\tau}{h^{2}}\left({ }_{j} u_{n+1, i}^{k+1}-{ }_{j} u_{n+1, i}^{k}-2\left({ }_{j} u_{n, i}^{k+1}-{ }_{j} u_{n, i}^{k}\right)+{ }_{j} u_{n, i-1}^{k+1}-{ }_{j} u_{n, i-1}^{k}\right)\right\}_{n, i=1}^{M-1} \\
-\left\{\frac{\tau}{h^{2}}\left({ }_{j} u_{n, i+1}^{k+1}-{ }_{j} u_{n, i+1}^{k}-2\left({ }_{j} u_{n, i}^{k+1}-{ }_{j} u_{n, i}^{k}\right)+{ }_{j} u_{n-1, i}^{k+1}-{ }_{j} u_{n-1, i}^{k}\right)\right\}_{n, i=1}^{M-1} \\
=\frac{u^{k}-u^{k-1}}{\tau}, k=m N+1, m=0,1, \ldots, k \geq 1 .
\end{gathered}
$$

Here $R, A, B$, and $C$ are $(M+1) \times(M+1) \times(M+1)$ given matrices and $\varphi\left({ }_{j-1} u^{k}, u^{k-N}\right)$ and $j u^{s}, s=k, k \pm 1$ are given $(M+1) \times(M+1) \times 1$ column vectors. Therefore, we will use the same algorithm as the one dimensional case. 
So, we have the initial value problem for the second order difference equation with respect to $k$ with matrix coefficients. From Equations (53) and (54) it follows that

$$
\begin{gathered}
{ }_{j} u^{k+1}=-A^{-1}\left(B_{j} u^{k}-C_{j} u^{k-1}+A^{-1} R \varphi^{k}\left({ }_{j-1} u^{k}, u^{k-N}\right)\right), 1 \leq k<\infty, \\
u^{k}=\left\{e^{-t_{k}} \sin x_{n} \sin x_{i}\right\}_{n, i=0}^{M},-N \leq k \leq 0, \\
{ }_{j} u^{k+1}=\psi\left(u^{k}, u^{k-1}\right), \quad k=m N+1, m=0,1, \ldots, k \geq 1 .
\end{gathered}
$$

Here, $\psi\left(u^{k}, u^{k-1}\right)$ is the given $(M+1) \times(M+1) \times 1$ column vector.

In computations the initial guess is chosen as ${ }_{0} u^{k}=\left\{\sin x_{n} \sin x_{i}\right\}_{n, i=0}^{M}$ and when the maximum errors between two consecutive results of iterative difference scheme (57) become less than $10^{-6}$, the iterative process is terminated. We present numerical results for different values of $N$ and $M$ and $u_{n, i}^{k}$ represent the numerical solutions of this difference scheme at $\left(t_{k}, x_{n}, x_{i}\right)$. The table is constructed for $N=M=20,40,80$ in $t \in[0,1], t \in[1,2], t \in[2,3]$, respectively and the errors are computed by the following formula

$$
m E_{M, M}^{N}=\max _{m N+1 \leq k \leq(m+1) N, 0 \leq n, i \leq M}\left|u\left(t_{k}, x_{n}, x_{i}\right)-{ }_{j} u_{n, i}^{k}\right| .
$$

As can be seen from table, these numerical experiments support the theoretical statements. The number of iterations and maximum errors are decreasing with the increase of grid points.

In Table 2, as we increase values of $M$ and $N$ each time starting from $M=N=30$ by a factor of 2 the errors in the first order of accuracy difference scheme decrease approximately by a factor of $1 / 2$. The errors presented in tables indicate the the time convergence order is one. This result fits with the theoretical results perfectly.

Table 2. The errors of difference scheme (57) (Number of the iteration $=j$ ).

\begin{tabular}{cccc}
\hline$m E_{M, M}^{N} /(N, M, M)$ & $(\mathbf{2 0 , 2 0 , 2 0 )}$ & $\mathbf{( 4 0 , 4 0 , 4 0 )}$ & $\mathbf{( 8 0 , 8 0 , 8 0 )}$ \\
\hline in $t \in[0,1]$ & $1.2517 \times 10^{-2}, j=4$ & $0.6554 \times 10^{-2}, j=4$ & $0.3348 \times 10^{-2}, j=4$ \\
\hline in $t \in[1,2]$ & $1.2517 \times 10^{-2}, j=3$ & $0.6556 \times 10^{-2}, j=3$ & $0.3348 \times 10^{-2}, j=3$ \\
\hline in $t \in[2,3]$ & $2.3678 \times 10^{-3}, j=2$ & $1.4306 \times 10^{-3}, j=2$ & $0.7870 \times 10^{-3}, j=2$ \\
\hline
\end{tabular}

\subsection{Conclusions and Our Future Plans}

1. In the present paper, the main theorem on the existence and uniqueness of a bounded solution of the initial value problem for a semilinear hyperbolic equation with time delay in a Hilbert space with the self adjoint positive definite operator is established. In applications, the existence and uniqueness of a bounded solution of four problems for semilinear hyperbolic equations with time delay are obtained. A first order of accuracy difference scheme for the numerical solution of the abstract problem is presented. The theorem on the existence and uniqueness of an uniformly bounded solution of this difference scheme with respect to $\tau$ is established. In applications, the existence and uniqueness of a uniformly bounded solutions with respect to time and space stepsizes of difference schemes for four semilinear partial differential equations with time delay are obtained. Numerical results for the solution of difference schemes for one and two dimensional semilinear delay hyperbolic equation are presented.

2. We are interested in studying uniformly boundedness of solutions of high order of accuracy difference schemes uniformly with respect to time stepsize of approximate solutions of this initial-value problem, in which bounded solutions were established without any assumptions in respect to the 
grid steps $\tau$ and $h$. We have not been able to establish such type of results for the solution of the very well-known second order difference scheme

$$
\left\{\begin{array}{l}
\frac{u^{k+1}-2 u^{k}+u^{k-1}}{\tau^{2}}+A u^{k}=f\left(t_{k}, u^{k}, \frac{u^{k-N}-u^{k-N-1}}{\tau}, u^{k-N}\right), \\
t_{k}=k \tau, \quad 1 \leq k<\infty, \quad N \tau=\omega, \\
\left(I+\tau^{2} A\right) \frac{u^{k+1}-u^{k}}{\tau}=\frac{2 u^{k}-3 u^{k-1}+u^{k-2}}{\tau}, k=m N, \quad m=1, \ldots, \\
u^{k}=\varphi\left(t_{k}\right), \quad t_{k}=k \tau, \quad-N \leq k \leq 0 .
\end{array}\right.
$$

Note that absolute stable two-step difference schemes of the high order of approximation for hyperbolic partial differential equations were presented and investigated in papers [11,54]. Applying methods of the present paper and papers $[11,54]$ we can establish the similar stability and convergence results of this paper for the solution of the absolute stable two-step difference schemes of high order of approximation for semilinear delay hyperbolic equations.

3. Investigate the uniform to-step difference schemes and asymptotic formulas for the solution of initial value perturbation problem

$$
\left\{\begin{array}{l}
\varepsilon^{2} u^{\prime \prime}(t)+A u(t)=f\left(t, u(t), u_{t}(t-w), u(t-w)\right), t>0 \\
u(t)=\varphi(t),-w \leq t \leq 0
\end{array}\right.
$$

for a semilinear delay hyperbolic equation in a Hilbert space $H$ with the self adjoint positive definite operator $A$ and with $\varepsilon \in(0, \infty)$ parameter multiplying the highest order derivative term.

In [31], the uniform difference schemes and asymptotic formulas for the solution of initial value perturbation problem for a linear hyperbolic equation in a Hilbert space with the self adjoint positive definite operator and with $\varepsilon \in(0, \infty)$ parameter multiplying the highest order derivative term were presented and investigated.

4. Investigate the initial value problem

$$
\left\{\begin{array}{l}
u_{t t}(t) d t+A u(t) d t=f\left(t, u(t), u_{t}(t-w), u(t-\omega)\right) d w_{t} \\
w_{t}=\sqrt{t} \xi, \quad \xi \in N(0,1), \quad t>0 \\
u(t)=0, \quad-\omega \leq t \leq 0
\end{array}\right.
$$

for a semilinear stochastic hyperbolic equation with time delay in a Hilbert space $H$ with the self adjoint positive definite operator $A$. Here, $w_{t}$ is a standard Wiener process given on the probability space $(Q, F, P)$.

Note that absolute stable difference schemes for stochastic linear hyperbolic equations in Hilbert spaces were presented and investigated in [30].

Finally, in paper [55], a Lie algebra approach is applied to solve an SIS model where infection rate and recovery rate are time-varying. The method presented here has been used widely in chemical and physical sciences but not in epidemic applications due to insufficient symmetries.

Author Contributions: Investigation, A.A. and D.A.

Funding: This research was funded by "Russian Foundation for Basic Research (RFBR) grant number 16-01-00450."

Acknowledgments: The publication has been prepared with the support of the "RUDN University Program 5-100" and dedicated in memory of Pavel Evseevich Sobolevskii. The authors are grateful to Francisco Rodríguez and reviewers of this paper for the useful comments and relevant references. 
Conflicts of Interest: The authors declare no conflict of interest.

\section{References}

1. Ardito, A.; Ricciardi, P. Existence and regularity for linear delay partial differential equations. Nonlinear Anal. 1980, 4, 411-414. [CrossRef]

2. Arino, A. Delay Differential Equations and Applications; Springer: Berlin/Heidelberg, Germany, 2006.

3. Bhalekar, S.; Patade, J. Analytic solutions of nonlinear with proportional delays. Appl. Comput. Math. 2016, 15, 331-345.

4. Blasio, G.D. Delay differential equations with unbounded operators on delay terms. Nonlinear Anal.-Theory Appl. 2003, 52, 1-18. [CrossRef]

5. Kurulay, G.; Ozbay, H. Design of first order controllers for a flexible robot arm with time delay. Appl. Comput. 2017, 16, 48-58.

6. Skubachevskii, A.L. On the problem of attainment of equilibrium for control-system with delay. Dokl. Akad. 1994, 335, 157-160.

7. Vlasov, V.V.; Rautian, N.A. Spectral Analysis of Functional Differential Equations; MAKS Press: Moscow, Russia, 2016. (In Russian)

8. Shang, Y. On the delayed scaled consensus problems. Appl. Sci. 2017, 7, 713. [CrossRef]

9. Atay, F.M. On the duality between consensus problems and Markov processes, with application to delay systems. Markov Process. Relat. Field 2016, 22, 537-553.

10. Lu, X. Combined iterative methods for numerical solutions of parabolic problems with time delays. Appl. Math. Comput. 1998, 89, 213-224. [CrossRef]

11. Ashyralyev, A.; Sobolevskii, P.E. A note on the difference schemes for hyperbolic equations. Abstr. Appl. Anal. 2001, 6, 63-70. [CrossRef]

12. Agirseven, D. Approximate solutions of delay parabolic equations with the Dirichlet condition. Abstr. Appl. Anal. 2012, 2012, 682752. [CrossRef]

13. Ashyralyev, A.; Agirseven, D. Stability of parabolic equations with unbounded operators acting on delay terms. Electron. J. Differ. Equ. 2014, 2014, 1-13.

14. Ashyralyev, A.; Agirseven, D. On source identification problem for a delay parabolic equation. Nonlinear Anal. Model. Control 2014, 19, 335-349. [CrossRef]

15. Ashyralyev, A.; Agirseven, D. Stability of delay parabolic difference equations. Filomat 2014, 28, 995-1006. [CrossRef]

16. Ashyralyev, A.; Agirseven, D. Well-posedness of delay parabolic equations with unbounded operators acting on delay terms. Bound. Value Probl. 2014, 126. [CrossRef]

17. Ashyralyev, A.; Agirseven, D. Well-posedness of delay parabolic difference equations. Adv. Differ. Equ. 2014, 2014. [CrossRef]

18. Ashyralyev, A.; Agirseven, D. On convergence of difference schemes for delay parabolic equations. Comput. Math. Appl. 2013, 66, 1232-1244. [CrossRef]

19. Ashyralyev, A.; Agirseven, D.; Ceylan, B. Bounded solutions of delay nonlinear evolutionary equations. J. Comput. Appl. Math. 2017, 318, 69-78. [CrossRef]

20. HenrÃ-quez, H.R.; Cuevas, C.; Caicedo, A. Almost periodic solutions of partial differential equations with delay. Adv. Differ. Equ. 2015, 2015. [CrossRef]

21. Hao, Z.P.; Fan, K.; Cao, W.R.; Sun, Z.Z. A finite difference scheme for semilinear space-fractional diffusion equations with time delay. Appl. Math. Comput. 2016, 275, 238-254. [CrossRef]

22. Liang, H. Convergence and asymptotic stability of Galerkin methods for linear parabolic equations with delays. Appl. Math. Comput. 2015, 264, 160-178. [CrossRef]

23. Bhrawy, A.H.; Abdelkawy, M.A.; Mallawi, F. An accurate Chebyshev pseudospectral scheme for multi-dimensional parabolic problems with time delays. Bound. Value Probl. 2015, 2015. [CrossRef]

24. Ismailov, Z.I.; Guler, B.O.; Ipek, P. Solvable time-delay differential operators for first order and their spectrums. Hacet. J. Math. Stat. 2016, 45, 755-764.

25. Piriadarshani, D.; Sengadir, T. Existence of solutions and semi-discretization for PDE with infinite delay. Differ. Equ. Appl. 2015, 7, 313-331. [CrossRef] 
26. Castro, M.A.; Rodriguez, F.; Cabrera, J.; Martin, J.A. Difference schemes for time-dependent heat conduction models with delay. Int. J. Comput. Math. 2014, 91, 53-61. [CrossRef]

27. Fattorini, H.O. Second Order Linear Differential Equations in Banach Spaces; Elsevier Science Publishing Company: Amsterdam, The Netherlands, 1985.

28. Goldstein, J.A. Semigroups of Linear Operators and Applications; Oxford Mathematical Monographs; The Clarendon Press Oxford University Press: New York, NY, USA, 1985.

29. Krein, S.G. Linear Differential Equations in Banach Space; American Mathematical Society: Providence, RI, USA, 1971.

30. Ashyralyev, A.; Akat, M. An approximation of stochastic hyperbolic equations: Case with Wiener process. Math. Appl. Sci. 2013, 36, 1095-1106. [CrossRef]

31. Ashyralyev, A.; Fattorini, H.O. On uniform difference-schemes for 2nd-order singular pertubation problems in Banach spaces. SIAM J. Math. Anal. 1992, 23, 29-54. [CrossRef]

32. Vasilev, V.V.; Krein, S.G.; Piskarev, S. Operator Semigroups, Cosine Operator Functions, and Linear Differential Equations; Itogi Nauki i Tekhniki Academy of Science USSR: Moscow, Russia, 1990.

33. Shang, Y. Non-hyperbolicity of random graphs with given expected degrees. Stoch. Models 2013, 29, 451-462. [CrossRef]

34. Shang, Y. Lack of Gromov-hyperbolicity in small-world networks. Cent. Eur. J. Math. 2012, 10. [CrossRef]

35. Ashyralyyeva, M.A.; Ashyraliyev, M. On the numerical solution of identification hyperbolic-parabolic problems with the Neumann boundary condition. Bull. Karaganda Univ.-Math. 2018, 91, 69-74. [CrossRef]

36. Ashyralyyeva, M.; Ashyraliyev, M. Numerical Solutions of Source Identification Problem for Hyperbolic-Parabolic Equations. In AIP Conference Proceedings; AIP Publishing: Melville, NY, USA, 2018; Volume 1997, p. 020048.

37. Zilal, D.; Ashyraliyev, M. Difference schemes for the semilinear integral-differential equation of the hyperbolic type. Filomat 2018, 32, 1009-1018.

38. Mohanty, R.K. An unconditionally stable finite difference formula for a linear second order one space dimensional hyperbolic equation with variable coefficients. Appl. Math. Comput. 2005, 165, 229-236. [CrossRef]

39. Mohanty, R.K. An operator splitting technique for an unconditionally stable difference method for a linear three space dimensional hyperbolic equation with variable coefficients. Appl. Math. Comput. 2005, 165, 549-557. [CrossRef]

40. Mohanty, R.K. An operator splitting method for an unconditionally stable difference scheme for a linear hyperbolic equation with variable coefficients in two space dimensions. Appl. Math. Comput. 2004, 152, 799-806. [CrossRef]

41. Piskarev, S. Stability of difference schemes in Cauchy problems with almost periodic solutions. Differentsial' nye Uravneniya 1994, 20, 689-695. (In Russian)

42. Piskarev, S. Principles of Discretization Methods III; Report ak -3410; Acoustic Institute, Academy of Science USSR: Moscow, Russia, 1986. (In Russian)

43. Sobolevskii, P.E.; Chebotaryeva, L.M. Approximate solution by method of lines of the Cauchy problem for an abstract hyperbolic equations. Vyssh. Uchebn. Zav. Mat. 1977, 5, 103-116. (In Russian)

44. Ashyralyev, A.; Pastor, J.; Piskarev, S.; Yurtsever, H.A. Second order equations in functional spaces: Qualitative and discrete well-posedness. Abstr. Appl. Anal. 2015, 2015, 1-63. [CrossRef]

45. Poorkarimi, H.; Wiener, J. Bounded solutions of non-linear hyperbolic equations with delay. In Proceedings of the VII International Conference on Non-Linear Analysis, Arlington, TX, USA, 28 July-1 August 1986; Volume 1, pp. 471-478.

46. Poorkarimi, H.; Wiener, J.; Shah, S.M. On the exponential growth of solutions to non-linear hyperbolic equations. Int. J. Math. Sci. 1989, 12, 539-546.

47. Shah, S.M.; Poorkarimi, H.; Wiener, J. Bounded solutions of retarded nonlinear hyperbolic equations. Bull. Allahabad Math. Soc. 1986, 1, 1-14.

48. Wiener, J. Generalized Solutions of Functional Differential Equations; World Scientific: Singapore, 1993.

49. Ashyralyev, A.; Agirseven, D. Bounded solutions of nonlinear hyperbolic equations with time delay. Electron. J. Differ. Equ. 2018, 2018, 1-15.

50. Ashyralyev, A.; Sobolevskii, P.E. New Difference Schemes for Partial Differential Equations; Birkhäuser Verlag: Basel, Switzerland, 2004. 
51. Ashyralyev, A. A survey of results in the theory of fractional spaces generated by positive operators. TWMS J. Pure Appl. Math. 2015, 6, 129-157.

52. Ashyralyev, A.; Sarsanbi, A. Well-posedness of a parabolic equation with involution. Numer. Funct. Anal. Optim. 2017, 38, 1295-1305. [CrossRef]

53. Sobolevskii, P.E. Difference Methods for the Approximate Solution of Differential Equations; Voronezh State University Press: Voronezh, Russia, 1975. (In Russian)

54. Ashyralyev, A.; Sobolevskii, P.E. Two new approaches for construction of the high order of accuracy difference schemes for hyperbolic differential equations. Discret. Dyn. Nat. Soc. 2005, 2005, 183-213. [CrossRef]

55. Shang, Y. A Lie algebra approach to susceptible-infected-epidemics. Electron. J. Differ. Equ. 2012, 233, 1-7.

(C) 2019 by the authors. Licensee MDPI, Basel, Switzerland. This article is an open access article distributed under the terms and conditions of the Creative Commons Attribution (CC BY) license (http://creativecommons.org/licenses/by/4.0/). 